\title{
High intensity cyclotrons for neutrino physics
}

\author{
Daniel Winklehner ${ }^{\mathrm{a}, *}$, Jungbae Bahng ${ }^{\mathrm{a}}$, Luciano Calabretta ${ }^{\mathrm{c}}$, Alessandra Calanna ${ }^{\mathrm{c}}$, Alok Chakrabarti ${ }^{\mathrm{d}}$, Janet Conrad ${ }^{\mathrm{a}}$, \\ Grazia D'Agostino ${ }^{c}$, Siddharta Dechoudhury ${ }^{\mathrm{d}}$, Vaishali Naik ${ }^{\mathrm{d}}$, Loyd Waites ${ }^{\mathrm{a}}$, Philip Weigel ${ }^{\mathrm{b}}$ \\ ${ }^{a}$ Massachusetts Institute of Technology, 77 Massachusetts Ave, Cambridge, MA 02139, USA \\ ${ }^{b}$ Drexel University, 3141 Chestnut Street, Philadelphia, PA 19104, USA \\ ${ }^{c}$ INFN, Via S. Sofia 62, 95123 Catania, Italy \\ ${ }^{d}$ VECC 83 HBNI, Sector-1, Block-AF, Bidhan Nagar, Kolkata 700064, India
}

\section{$\infty$ Abstract}

In recent years, the interest in high intensity proton beams in excess of several milli-amperes has risen. Potential applications are in neutrino physics, materials and energy research, and isotope production. Continuous wave proton beams of five to ten milli-amperes are now in reach due to advances in accelerator technology and through improved understanding of the beam dynamics. As an example application, we present the proposed IsoDAR experiment, a search for so-called sterile neutrinos and non-standard interaction using the KamLAND detector located in Japan. We present updated sensitivities for this experiment and describe in detail the design of the high intensity proton driver that uses several novel ideas. These are: accelerating $\mathrm{H}_{2}^{+}$instead of protons, directly injecting beam into the cyclotron via a radio frequency quadrupole, and carefully matching the beam to achieve so-called vortex motion. The preliminary design holds up well in PIC simulation studies and the injector system is now being constructed, to be commissioned with a $1 \mathrm{MeV} / \mathrm{amu}$ test cyclotron.

Keywords: Cyclotrons, High Current, PIC, RFQ

\section{Introduction}

High intensity proton beams are used very successfully in spallation neutron sources like the Spallation Neutron Source (SNS) at Oak Ridge National Laboratory (ORNL) 1], the Materials and Life Science Experimental Facility (MLF) at the Japan Proton Accelerator Research Complex (J-PARC) [2], and the Swiss spallation neutron source SINQ at the Paul Scherrer Institute (PSI) 3]. In addition to neutrons these spallation targets can also produce kaons, pions, muons, and neutrinos to be used in particle physics experiments (e.g. [4]). With the exception of PSI, these facilities use linear accelerators to produce high intensity proton beams, which are costly and require significant space. Recent developments in accelerator technology and improved understanding of the beam dynamics in high intensity cyclotrons have made these circular particle accelerators an attractive candidate for new high intensity proton drivers. Currently, PSI holds the record of highest power continuous wave $(\mathrm{cw})$ proton beam from a cyclotron with $2.2 \mathrm{~mA}$ at $590 \mathrm{MeV}$ energy, which amounts to $\approx 1.3$ MW of beam power [5]. In this coupled cyclotron system, so-called vortex motion has been observed experimentally and been reproduced in Particle-In-Cell (PIC) simulations 6. 7]. Here, the external focusing forces of the isochronous cyclotron combined with space-charge cause a spiraling of the bunch that can lead to longitudinal focusing. We can utilize this effect to accelerate even higher beam currents as will be discussed in this paper. Some proposed experiments and potential applications of high intensity proton beams are listed in Table 1 together with current and energy requirements.

Table 1: A few potential uses for high current proton beams. (ADSR - Accelerator Driven Sub-critical Reactors, ADS - Accelerator Driven Systems)

\begin{tabular}{llll}
\hline Experiments & Type & Current & Energy \\
\hline \hline IsoDAR [8, 9] & neutrino exp. & $10 \mathrm{~mA}$ & $60 \mathrm{MeV}$ \\
DAE $\delta$ ALUS 10, 11] & neutrino exp. & $10 \mathrm{~mA}$ & $800 \mathrm{MeV}$ \\
ADSR [1, 13, & energy & $10 \mathrm{~mA}$ & $<1.2 \mathrm{GeV}$ \\
ADS 14] 15, & energy & $4-120 \mathrm{~mA}$ & $<1.2 \mathrm{GeV}$ \\
Isotopes 16, 17, & medicine & $<2 \mathrm{~mA}$ & $3-70 \mathrm{MeV}$ \\
\hline
\end{tabular}

After motivating the need for high intensity proton beams by using one of the given examples (the proposed neutrino experiment IsoDAR) in the following subsection, we will describe in detail the building blocks of a cyclotronbased proton driver in Section 2 together with the design choices that make such a system feasible. In Section 3 we will then present simulations of the individual parts, followed by a discussion of the next steps towards a running system in Section 4 .

\footnotetext{
* Corresponding author

Email address: winklehn@mit.edu (Daniel Winklehner)
} 


\subsection{An Example: The IsoDAR Experiment}

IsoDAR (Isotope Decay At Rest) is a novel, pure $\bar{\nu}_{e}$ source under development that makes use of a cyclotronaccelerated beam delivered to a decay-at-rest target. A high-intensity $\mathrm{H}_{2}^{+}$ion source feeds a $60 \mathrm{MeV} / \mathrm{amu}$ cyclotron. The beam is electron-stripped after extraction and transported to a ${ }^{9}$ Be target, producing neutrons. The neutrons enter $\mathrm{a} \geq 99.99 \%$ isotopically pure ${ }^{7} \mathrm{Li}$ sleeve, where neutron capture results in ${ }^{8} \mathrm{Li}$. The ${ }^{8} \mathrm{Li}$ undergoes $\beta$ decayat-rest, producing an isotropic, pure $\bar{\nu}_{e}$ flux. Pairing this very high-intensity $\bar{\nu}_{e}$ source with a detector that contains hydrogen allows for the inverse beta decay (IBD) interaction, $\bar{\nu}_{e}+p \rightarrow e^{+}+n$, and $\bar{\nu}_{e^{-}} e^{-}$elastic scattering (ES) processes.

Our goal in developing IsoDAR was to "think outside of the box" on neutrino sources for underground science. We are producing a cheaper, more compact and purer source than existing neutrino sources, with flexibility to install the source underground at most laboratories.

Our initial proposal is to install IsoDAR near to the KamLAND detector. The first proposal for the layout of the IsoDAR facility placed the source at $16 \mathrm{~m}$ from the center of the KamLAND detector and assumed an energy resolution of $6.5 \% / \sqrt{E}$ [9]. Recently, two potential improvements to this plan have been put forward. First, we have identified a new, potentially closer targeting site [18], at $12.5 \mathrm{~m}$, increasing rates by $\times 1.6$. Should this new location be employed, the additional statistics can allow us to either reach our physics goals more quickly or to run the cyclotron at $6 \mathrm{~mA}$ of protons $\left(3 \mathrm{~mA} \mathrm{H}_{2}^{+}\right)$rather than $10 \mathrm{~mA}$ of protons $\left(5 \mathrm{~mA} \mathrm{H}_{2}^{+}\right)$for the original time period. Running with $6 \mathrm{~mA}$ of protons is preferred to reduce operations cost and risk. Second, KamLAND is proposing an upgrade which will improve the resolution of the detector to $3 \% / \sqrt{E}$, which benefits IsoDAR physics.

Because IsoDAR is relatively inexpensive, one can envision many such sources in the future. For example, the JUNO collaboration has worked with us on potentially bringing IsoDAR to their detector after reactor running [19].

\subsection{Examples of the Physics of IsoDAR}

In this section, we describe two examples of the IsoDAR physics program in detail. IsoDAR also makes other beyond Standard Model searches, nuclear physics measurements and provides calibration for KamLAND.

\subsubsection{Sterile Neutrino Search}

Interest in light sterile neutrinos has arisen from anomalies observed in a wide range of short-baseline (SBL) experiments employing neutrinos and antineutrinos of different flavors and different energies [20, 21, 22, 23, 24, 25, 26]. However, other experiments potentially sensitive to sterile neutrinos have observed null results [27, 28, 29, 30, 31, 32, 33, 34. This limits models that describe the anomalies. One such model that introduces one additional sterile neutrino (" $3+1$ ") depends on four BSM parameters,
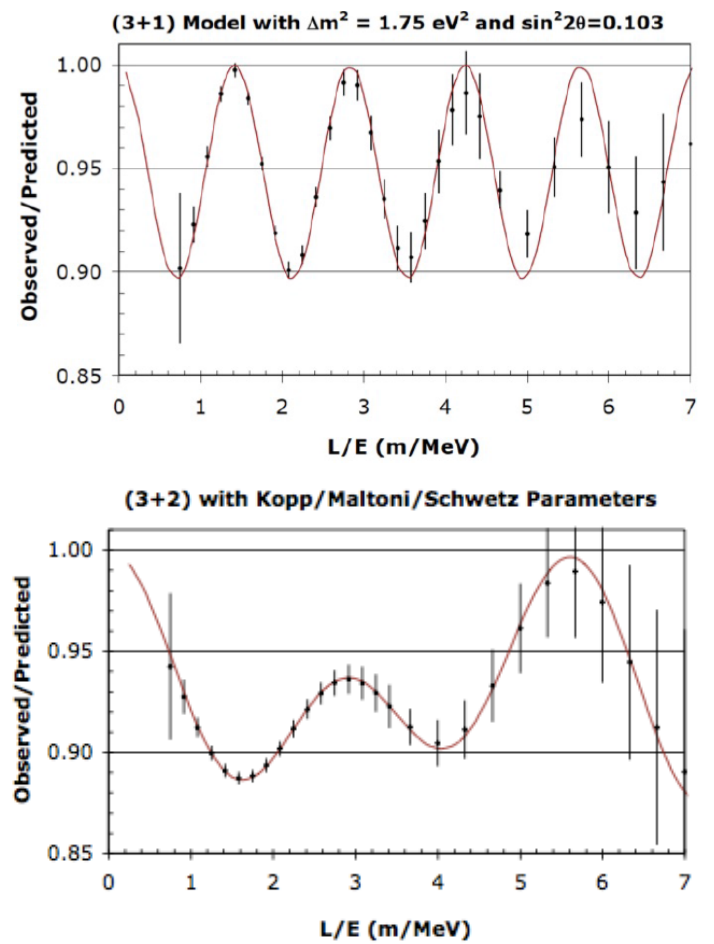

Figure 1: IsoDAR@KamLAND $L / E$ dependence, 5 years of running, for one (upper plot) and two (lower plot) sterile neutrinos. Solid curve is the oscillation probability with no smearing in the reconstructed position and energy and the data points with error bars are from simulated events including smearing from reconstruction for the original target location and detector (assumes present resolution, and target at $16 \mathrm{~m}$ ).

$\sin ^{2} 2 \theta_{e e}, \sin ^{2} 2 \theta_{\mu \mu}, \sin ^{2} 2 \theta_{\mu e}$, and $\Delta m^{2}$. The first three are the mixing angles measured in three types of oscillation experiments: $\nu_{e}$ disappearance, $\nu_{\mu}$ disappearance and $\nu_{\mu} \rightarrow \nu_{e}$ appearance. These are constructed of two matrix elements, $\left|U_{e 4}\right|^{2}$ and $\left|U_{\mu 4}\right|^{2}$, hence they are interdependent. The fourth parameter, the squared mass splitting between the mostly sterile and mostly active states must be consistent for all three types of oscillations.

Global fits that include all of the SBL data sets show a marked improvement in $\Delta \chi_{\text {null-min }}^{2} / \Delta$ dof of $50.61 / 4$ for a $3+1$ model 35. However, the appearance-only and disappearance-only allowed regions show poor overlap in allowed parameters [36]. This well-known tension is one of the primary reasons scientists question the sterile neutrino model.

IsoDAR was developed to allow a sterile neutrino search that can resolve the confusing situation of anomalies in the electron-flavor sector. IsoDAR provides a very wellunderstood flux and a highly sensitive method to search for sterile neutrinos through reconstruction of the $L / E$ dependence of the neutrino oscillation process, commonly referred to as an "oscillation wave," shown in Figure 1.

The IsoDAR 3-year, $5 \sigma$ expectations for our baseline design (red) 9] and for equal statistics at the new, closer site, with the detector upgrade (blue) [18] are shown in 


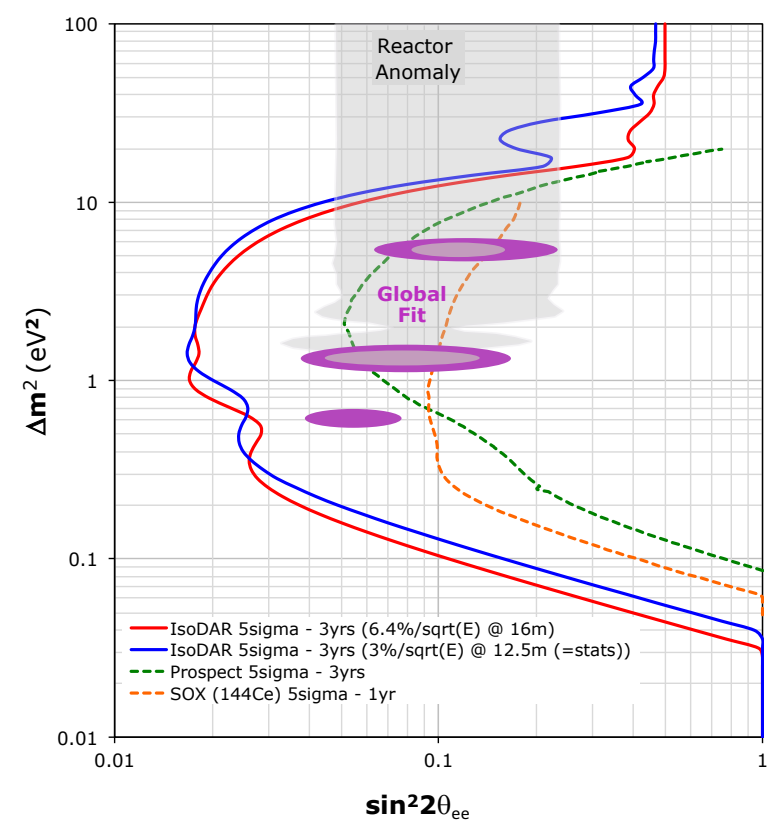

Figure 2: Isodar@KamLAND at original site (red), new proposed site with equal statistics (blue), and in comparison to other experiments.

Figure 2, compared to other proposed experiments (green, orange) 37, 38. IsoDAR@KamLAND addresses the reactor anomaly [23, and fully covers of all allowed islands from the $3+1$ global fit (magenta) 39. Figure 1 shows the $L / E$ distribution for two potential signals. The wellmeasured oscillation wave differentiates models with one sterile neutrino (left) and two sterile neutrinos (right) [8].

The well-understood ${ }^{8} \mathrm{Li}$ flux is a great advantage for IsoDAR compared with the ongoing reactor experiments. Reactor fluxes have two important issues that the IsoDAR flux avoids. The first is an excess in the reactor spectrum at $5 \mathrm{MeV}$ that is seen in most [40, 41, 42, but not all [43] cases, for which the source is unknown. Second, although Daya Bay has indicated the reactor anomaly may depend on fuel (U vs. $\mathrm{Pu}$ ) 44, three independent analyses [45, 46, 47] reach the conclusion that even after the new Daya Bay result, the reactor anomaly remains at $>2 \sigma$. IsoDAR does not face these questions.

\subsubsection{NSI Search Through Precision Electroweak Tests}

The search for indications of NSIs is a second example of the outstanding new physics opened by the IsoDAR source. This makes use of the fact that, running with KamLAND, the experiment will collect the largest sample of $\bar{\nu}_{e}$ ES events observed to date 48. Approximately 2600 ES events will be collected at KamLAND above a $3 \mathrm{MeV}$ visible energy threshold over a 5 year run-a factor of five above existing $\bar{\nu}_{e}$ samples [48]. The improved resolution of KamLAND may allow us to lower this threshold and increase statistics further. The ES rate can be normalized using the well-understood, very-high-statistics IBD events. Ref. 48 describes how the ES cross section can be used

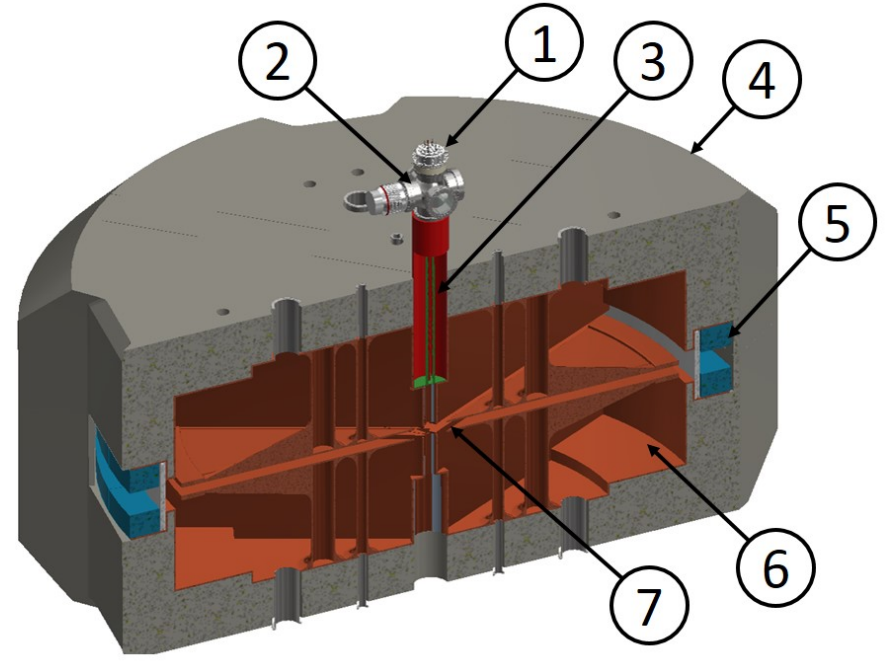

Figure 3: Conceptual layout of the compact cyclotron driver for high intensity $60 \mathrm{MeV} / \mathrm{amu} \mathrm{H}_{2}^{+}$beams. 1. Ion source, 2. diagnostic and pumping box, 3. Radio Frequency Quadrupole (RFQ) injector, 4. Compact cyclotron yoke, 5. Compact cyclotron coils, 6. Accelerating RF electrodes ("Dees"), 7. Central region.

to measure the weak couplings, $g_{V}^{\nu_{e} e}$ and $g_{A}^{\nu_{e} e}$. Deviation of these couplings could explain the NuTeV anomaly [49. These couplings can be recast in terms of the left and right handed couplings that may be modified by NSI's: $g_{L}^{S M} \rightarrow g_{L}^{S M}+\varepsilon_{e e}^{e L}$ and/or $g_{R}^{S M} \rightarrow g_{R}^{S M}+\varepsilon_{e e}^{e R}$. IsoDAR's capability to constrain NSI's is as strong as the combined present data sets, and is orthogonal because this is a pure antineutrino sample.

\section{Accelerator Design}

As mentioned in the introduction, in order for IsoDAR@KamLAND to be decisive in 5 years, a current of $10 \mathrm{~mA}$ of protons on target $(6 \mathrm{~mA}$ in the alternate location) is necessary. This is roughly a factor 4 more than world-record holder PSI Injector II has delivered so far. The main limitation being space-charge (the de-focusing self-fields of the beam resulting from Coulomb-repulsion between the individual particles). Space charge effects are most pronounced at low energies and high current densities, i.e. in the Low Energy Beam Transport line (LEBT) connecting ion source and accelerator, the injection into the accelerator, and during the first few turns in the cyclotron, until higher beam energy has been reached. In order to mitigate these detrimental space charge effects, we propose to accelerate $\mathrm{H}_{2}^{+}$ions instead of protons and, at the same time, increase the injection energy of the beam to $35 \mathrm{keV} / \mathrm{amu}$. Using $\mathrm{H}_{2}^{+}$allows the use of a stripper foil after extraction from the cyclotron to remove the binding electron, thereby doubling the electrical beam current. This reduces our requirement to $5 \mathrm{~mA}$ of $\mathrm{H}_{2}^{+}(3 \mathrm{~mA}$ in the alternate location). As will be explained in Section 2.3.5. using $\mathrm{H}_{2}^{+}$leads to a fairly large spiral inflector (the elec- 
trostatic device bending the beam from the axial direction onto the cyclotron median plane).

Another limiting factor is the phase acceptance of the cyclotron. Typically, for isochronous cyclotrons this is $\pm 10^{\circ}$ around the synchronous phase $\Phi_{\mathrm{S}}$. Our preliminary beam dynamics studies for the $60 \mathrm{MeV}$ /amu cyclotron have shown that the vortex effect can lead to a matched beam in later stages of acceleration even if the injected beam is as long as $\pm 20^{\circ}$, if carefully placed collimators are used to clean up beam halo in the first 10 turns (see [50, 51]). Even so, a significant portion of the injected beam will be lost. Our solution is to use a Radio Frequency Quadrupole (RFQ) to aggressively pre-bunch the beam. In order to bring the RFQ as close as possible to the central region of the cyclotron (injection point), it will be embedded partially in the iron yoke of the cyclotron. The conceptual layout of the proton driver is shown in Figure 3 . The main components of the driver are:

1. The ion source

2. The RFQ injector

3. The spiral inflector

4. The cyclotron

In order to demonstrate the $\mathrm{H}_{2}^{+}$production and RFQ bunching/injection capabilities, an NSF funded project is currently on the way at MIT, the $R F Q$-Direct Injection Project (RFQ-DIP), which combines items $1-3$ with a $1 \mathrm{MeV} / \mathrm{amu}$ test cyclotron. RFQ-DIP will also double as the actual ion source and RFQ for IsoDAR.

In the following subsections, we describe the individual components of the proton driver in more detail. For the full cyclotron design, we will mostly refer to previous publications (references given in Section 2.3 and will focus on the central region and the RFQ-DIP $1 \mathrm{MeV} / \mathrm{amu}$ test cyclotron design. However, these considerations are directly applicable in the full $60 \mathrm{MeV} / \mathrm{amu}$ machine.

\subsection{Ion source design}

In order to produce the required DC current of $\mathrm{H}_{2}^{+}(10-$ $15 \mathrm{~mA}$ ), a new multicusp ion source (MIST-1) has been constructed at MIT and is currently under commissioning. The new source is based on a design by Lawrence Berkeley National Laboratory dedicated to the production of $\mathrm{H}_{2}^{+}$over protons which demonstrated combined current densities of $50 \mathrm{~mA} / \mathrm{cm}^{2}$ and an $\mathrm{H}_{2}^{+}$fraction greater than $80 \%$ 52].

\subsubsection{Ion source design parameters}

A detailed description of the MIST-1 ion source can be found elsewhere [9, 53. In short, a plasma is confined by permanent magnets $\left(\mathrm{Sm}_{2} \mathrm{Co}_{17}\right)$, electrons are produced by a filament and are accelerated by an externally applied electric field of order $100 \mathrm{~V}$. Due to the short source and the plasma being produced close to the extraction region, there is not enough time for dissociation to occur, favoring $\mathrm{H}_{2}^{+}$over proton production. A half-section view of the ion source can be seen in Figure 4 and the main parameters are listed in Table 2

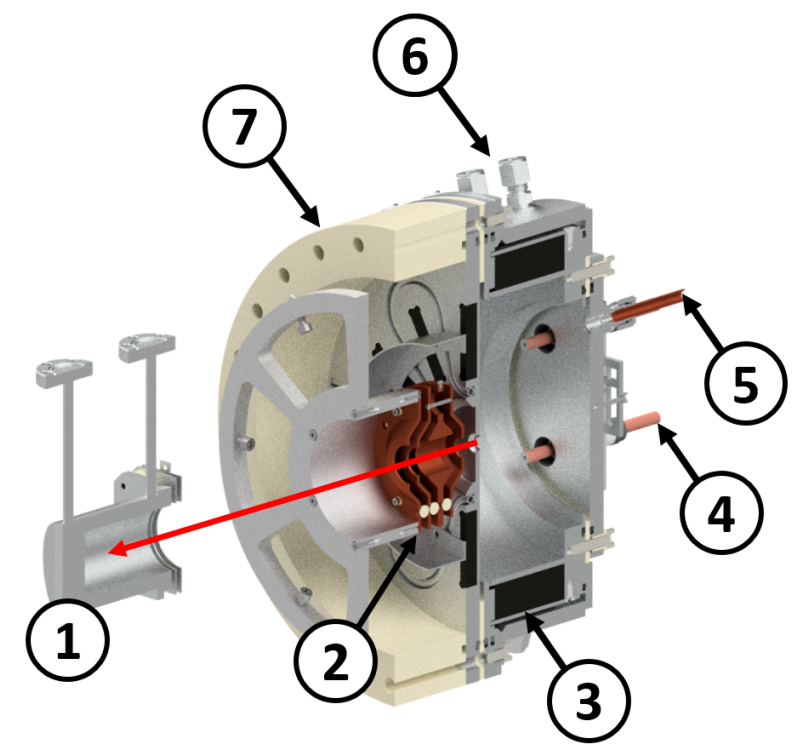

Figure 4: Cut view of the MIST-1 ion source. 1. Faraday cup, 2. Extraction System, 3. Permanent magnets $\left(\mathrm{Sm}_{2} \mathrm{Co} 17\right)$, 4. Filament feedthroughs, 5. Gas inlet, 6. Water cooling fittings, 7. Alumina insulator ring.

\subsubsection{Preliminary commissioning results}

In the first commissioning phase, a thinner $(0.4 \mathrm{~mm}$ diameter) pure tungsten filament was used instead of the nominal filament described in the previous section. Currents were measured in a Faraday cup right after the extraction system (see Figure 4), thus not allowing species separation. All reported currents are total extracted currents. During the accumulated run time of $\approx 30$ hours, the source showed good stability for about 4 hours at a

Table 2: MIST-1 ion source parameters.

\begin{tabular}{ll}
\hline Parameter & Value (nominal) \\
\hline Plasma chamber length & $6.5 \mathrm{~cm}$ \\
Plasma chamber diameter & $15 \mathrm{~cm}$ \\
Permanent magnet material & $\mathrm{Sm}_{2} \mathrm{Co}_{17}$ \\
Permanent magnet strength & $1.05 \mathrm{~T}$ on surface \\
Front plate magnets & 12 bars (star shape) \\
Radial magnets & 12 bars \\
Back plate magnets & 4 bars, 3 parallel rows \\
Front plate cooling & embedded steel tube \\
Back plate cooling & embedded copper tube \\
Chamber cooling & water jacket \\
Water flow (both) & $(1.5 \mathrm{l} / \mathrm{min})$ \\
Filament feedthrough cooling & air cooled heat sink \\
Filament material & $98 \% \mathrm{~W}, 2 \% \mathrm{Th}$ \\
Filament diameter & $\approx 1.5 \mathrm{~mm}$ \\
Discharge voltage & max. $150 \mathrm{~V}$ \\
Discharge current & max. $24 \mathrm{~A}$ \\
Filament heating voltage & max. $8 \mathrm{~V}$ \\
Filament heating current & max. $100 \mathrm{~A}$ \\
\hline
\end{tabular}




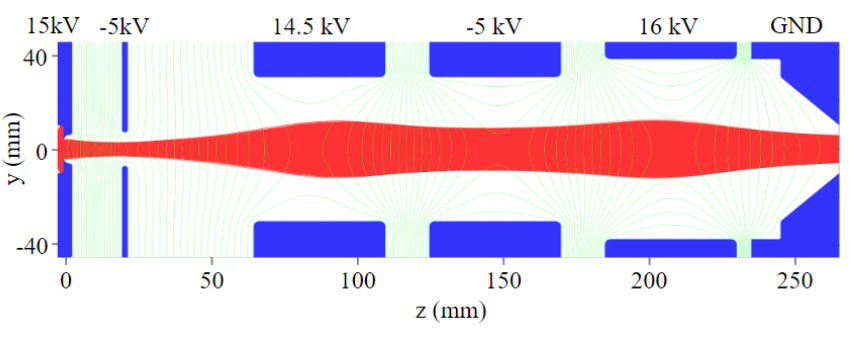

Figure 5: 2D cut view from center of extraction system of ion beam (red) moving through the extraction system before being transferred into the RFQ.

time, reaching a maximum current density of $16 \mathrm{~mA} / \mathrm{cm}^{2}$ (4.6 $\mathrm{mA}$ total) [54]. While at this point the commissioning results are preliminary, we are confident that our source will have similar species ratios $\left(\mathrm{p}^{+}: \mathrm{H}_{2}^{+}: \mathrm{H}_{3}^{+} \approx 1: 8: 1\right)$ as previous similar sources 52 . A paper on the final commissioning results using a short analysis beam line with dipole magnet for mass separation is forthcoming.

\subsubsection{Extraction system}

The extraction system serves two important purposes: initial shaping of the beam, and focusing the beam into the RFQ. The extraction system designed for the ion source commissioning (item 2 in Figure 4 ) is very short and not able to serve in the second function. For the final IsoDAR system and the intermediate RFQ-DIP, a new extraction system is being designed. A cross-sectional view is shown in Figure 5 The electrodes (from left to right) are: plasma electrode $(+15 \mathrm{kV})$, puller electrode $(-3$ to $15 \mathrm{kV})$, lens element $1(+10$ to $+15 \mathrm{kV})$, lens element 2 $(-3$ to $-15 \mathrm{kV})$, lens element $3(+10$ to $+15 \mathrm{kV})$, and RFQ entrance flange (ground). The extraction system was modeled using the well-established IBSIMU code [55]. IBSIMU calculates the ion trajectories in an iterative process, taking into account the (quasi-neutral) ion source plasma, the external fields generated by the electrodes, and the beam's self-fields (space charge). In the final design, lens elements 1 and 3 will be segmented (given diagonal slits similar to Ref. 56]) to allow small angle steering in both horizontal and vertical direction. A simplified picture of this scheme is shown in Figure 6 .

\section{2. $R F Q$ Design}

In order to improve injection efficiency into a compact isochronous cyclotron, the maximum possible prebunching is desired, as the accelerator can only accept beam into a phase window of $20-40^{\circ}$ An RFQ provides over $90 \%$ bunching efficiency, up to $100 \%$ transmission efficiency, and acts as a pre-accelerator. Unwanted ion species like protons and $\mathrm{H}_{3}^{+}$are lost to the electrodes and the cavity wall. In the following subsections the preliminary design of the IsoDAR/RFQ-DIP RFQ is discussed. A first start-to-end simulation from ion source extraction through the RFQ and spiral inflector, up to acceleration to $1 \mathrm{MeV} / \mathrm{amu}$ in the cyclotron is presented in Section 3

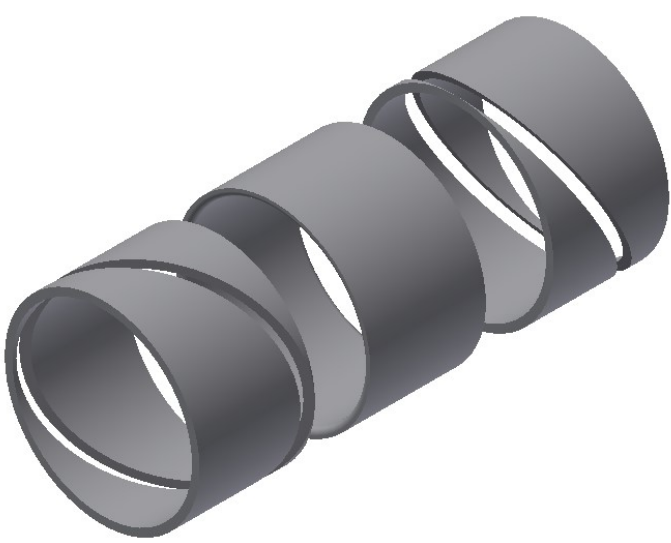

Figure 6: Cartoon picture of the proposed segmentation of lens elements 1 and 3 to allow small angle steering for additional control over matching the beam into the RFQ.

\subsubsection{RFQ design considerations}

The design requirements for the RFQ are listed in Table 3. These requirements for the IsoDAR/RFQ-DIP RFQ are driven by the following considerations: A compact cyclotron was chosen as the main accelerator due to space restrictions near underground neutrino detectors. Thus axial injection is the only viable solution to inject beam into the cyclotron. To avoid unnecessary beam spreading between RFQ exit and spiral inflector entrance, the RFQ must be brought as close as possible to the cyclotron median plane. The frequency must be matched to the cyclotron operation frequency $(32.8 \mathrm{MHz})$, the size (length, diameter) follows directly from the necessity to embed the RFQ partially in the cyclotron's iron yoke. The input energy $(7.5 \mathrm{keV} / \mathrm{amu})$ is minimized to reduce length (cell length $\left.=v_{z} / c \cdot \lambda\right)$ and the output energy $(35 \mathrm{keV} / \mathrm{amu})$ is the maximum energy that can safely be injected through a large spiral inflector. As will be discussed in more detail later, in order to achieve the comparably low operation frequency of $32.8 \mathrm{MHz}$ while maintaining the required small diameter, a split-coaxial design has been employed. In order to reduce energy spread and longitudinal beam size at the matching point (first accelerating gap in the cyclotron central region) a re-bunching cell is added to the RFQ.

We followed the usual cycle of beginning with a first order physics/beam dynamics design using the simulation software PARMTEQM developed at Los Alamos National

Table 3: Requirements for the RFQ design.

\begin{tabular}{|c|c|c|}
\hline Parameter & Value (nominal) & Unit \\
\hline Frequency & 32.8 & $\mathrm{MHz}$ \\
\hline Input energy & 15 & $\mathrm{keV}$ \\
\hline Length & $<1500$ & $\mathrm{~mm}$ \\
\hline Particle & $\mathrm{H}_{2}^{+}$ & $(\mathrm{q} / \mathrm{A}=1 / 2)$ \\
\hline Output energy & 70 & $\mathrm{keV}$ \\
\hline Diameter & $<300$ & $\mathrm{~mm}$ \\
\hline
\end{tabular}



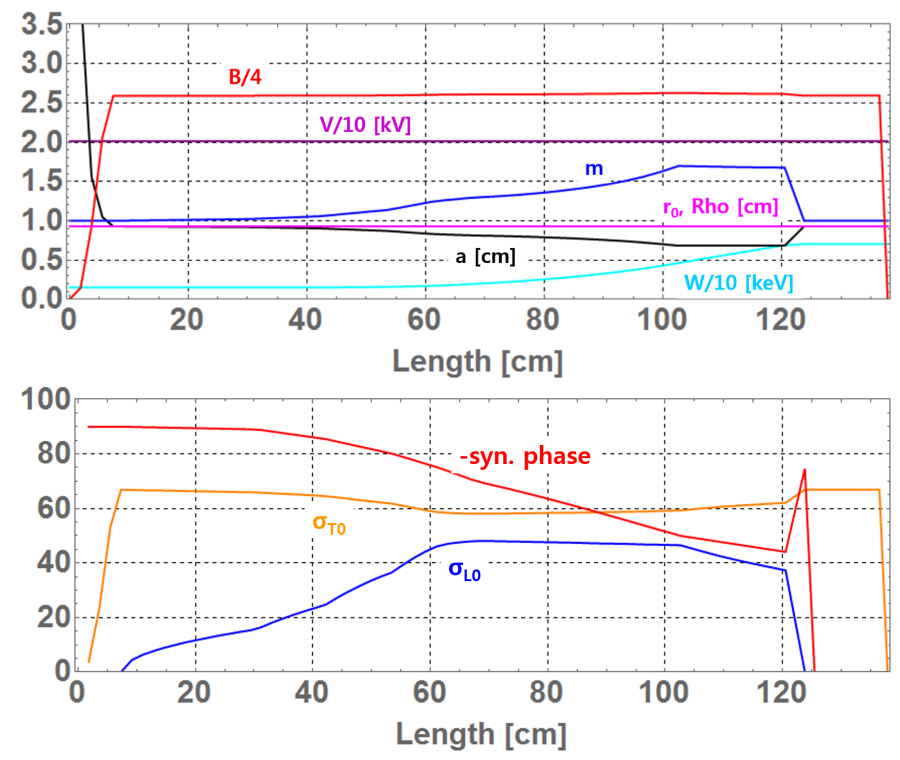

Figure 7: RFQ Design parameters versus cell number.

Laboratory (LANL) [57, followed by a 3D Particle-InCell (PIC) simulation using a fieldmap calculated from the PARMTEQM vane/rod shapes. For the PIC calculations we used TRACK [58] and fields were calculated by a Finite Elements Method (FEM) in CST Microwave Studio [59] after modeling the vanes in Autodesk Inventor.

\subsection{2. $R F Q$ beam dynamics design with PARMTEQM}

In addition to the fixed requirements of Table 3 , it is desirable have a constant vane voltage along the RFQ for added ease of machining. The detailed design results of the IsoDAR RFQ are listed in Table 4 and plotted in Figure 7 This preliminary RFQ design features a constant vane voltage of $20.14 \mathrm{kV}$, vane-tip curvature $\rho$ of $9.30 \mathrm{~mm}$ and mid-radius $r_{0}$ of $9.30 \mathrm{~mm}$. This gives a comparably simple structure with easy operation and easy tuning as well as a small longitudinal emittance of $40.24 \mathrm{keV}-\mathrm{deg}$ and a high transmission rate of $97.27 \%$. Since modulation and synchronous phase in the low energy region are a key

Table 4: RFQ design parameters.

\begin{tabular}{lrl}
\hline Parameter & Value & Unit \\
\hline Frequency & 32.80 & $\mathrm{MHz}$ \\
Length & 1378.69 & $\mathrm{~mm}$ \\
Transmission rate & 97.27 & $\%$ \\
$\epsilon_{\text {transv.,n,4rms }}$ & 1.37 & $\pi$-mm-mrad \\
a, min. vane-tip & 6.83 & $\mathrm{~mm}$ \\
r0, mid-radius & 9.30 & $\mathrm{~mm}$ \\
Vane voltage & 20.14 & $\mathrm{kV}$ \\
$\epsilon_{\text {long.,1rms }}$ & 40.24 & $\mathrm{keV}$-deg \\
$\rho$, vane-tip curvature & 9.30 & $\mathrm{~mm}$ \\
Octupole term & 0.07 & \\
No. of cells & 58 & \\
\hline
\end{tabular}

parameter to determine longitudinal emittance, adiabatic varied modulation and synchronous phase were applied as shown in Figure 7 to obtain a high transmission rate and small longitudinal emittance. In addition, we smoothly varied parameters in the shaper and gentle buncher sections to avoid transverse emittance blow-up at the end of the bunching section due to strong defocussing term.

\subsubsection{RFQ geometrical cavity design}

In general, a four-vane cavity type has a simple shape and high shunt impedance. Unfortunately, for low frequencies the transverse dimension gets prohibitively large. As mentioned above, for this project, cavity size is critical. Instead, a split-coaxial cavity design has been proposed and developed to obtain a small diameter at low operation frequency. In the split-coaxial cavity design, two opposite vanes are connected to one of the end-plates and the two other vanes (again opposite vanes) are connected to the other end-plate (this is depicted in Figure 8). The smaller transverse size in the split-coaxial RFQ cavity is due to the special magnetic flux structure 60. In addition to the lower RF frequency, this also provides high stability of the acceleration field since the magnetic field encompasses all four vanes as a coaxial waveguide. The geometrical cavity parameters for our split-coaxial RFQ cavity are defined as shown in Figure 8 and listed in Table 5. The RF frequency for this structure has been calculated to be 32.64 $\mathrm{MHz}$ using CST Microwave Studio.

\subsubsection{RFQ re-buncher cell design}

Finally, to achieve minimum energy spread and beam length at the matching point (first accelerating cavity of the cyclotron), a re-buncher cell is added to the RFQ, positioning the longitudinal beam focus about $40 \mathrm{~cm}$ after the RFQ exit. Matching to the cyclotron will be discussed in more detail in Section 2.3.1. The design parameters of the re-buncher are length, modulation, and position. The required length of re-buncher is calculated as $78.94 \mathrm{~mm}$

Table 5: RFQ cavity geometrical parameters. Select parameters are also shown in Figure 8

\begin{tabular}{lrl}
\hline Parameter (description) & Value & Unit \\
\hline R (cavity radius) & 120.00 & $\mathrm{~mm}$ \\
r (electrode radius) & 9.30 & $\mathrm{~mm}$ \\
d (electrode distance) & 18.60 & $\mathrm{~mm}$ \\
g1 (gap vert. vane $\leftrightarrow$ end plate) & 25.62 & $\mathrm{~mm}$ \\
g2 (gap horz. vane $\leftrightarrow$ end plate) & 8.35 & $\mathrm{~mm}$ \\
p (vane skirt position) & 60.0 & $\mathrm{~mm}$ \\
l1 (horizontal vane length) & 1353.07 & $\mathrm{~mm}$ \\
l2 (vertical vane length) & 1370.34 & $\mathrm{~mm}$ \\
L (cavity length) & 1378.69 & $\mathrm{~mm}$ \\
t (cavity thickness) & 20.0 & $\mathrm{~mm}$ \\
s (vane skirt max. thickness) & 30.0 & $\mathrm{~mm}$ \\
h (vane skirt min. thickness) & 10.0 & $\mathrm{~mm}$ \\
\hline
\end{tabular}




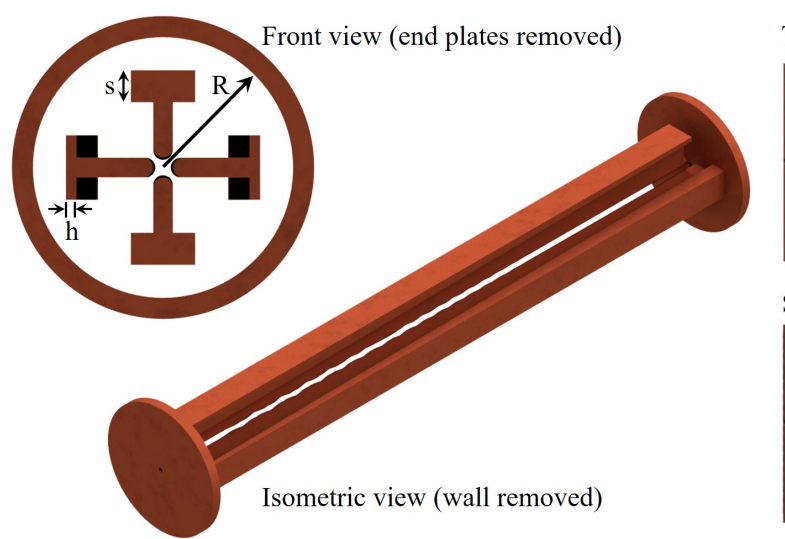

Top view (wall and vertical vanes removed) $\quad$ L

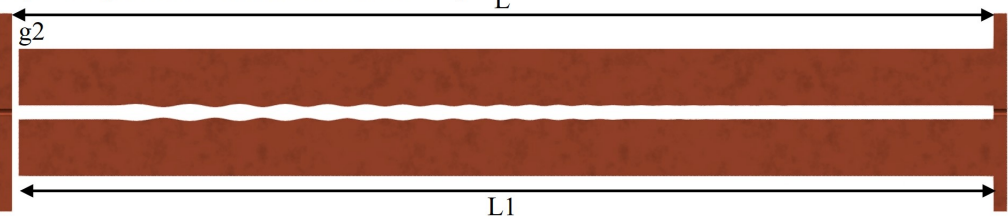

Side view (wall and horizontal vanes removed)

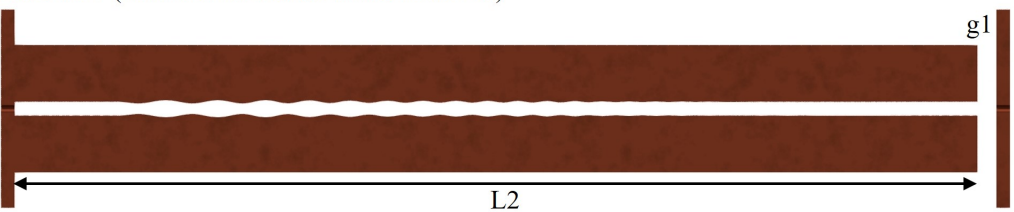

Figure 8: CAD renderings of the RFQ without re-bunching cell. The beam enters from the right. The most important geometry parameters are indicated and listed in Table 5 In the front and isometric views, the tapered skirts are visible that are used to achieve the design frequency and add additional stability against droop.

based on the cell length, $\beta \cdot \lambda$. The modulation is determined to match the longitudinal focal length between RFQ and cyclotron. The position of the re-buncher in the RFQ is determined empirically to find the optimum position with respect to phase and length of the bunch. The re-buncher uses a trapezoidal structure instead of a sinusoidal one to obtain an efficient field distribution and field separation from RFQ field, as shown in Figure 9. The rebuncher is included in the CAD modeling of the vanes in Inventor.

\subsection{Cyclotron Design}

In the following subsections, we will briefly discuss the peculiarities of high intensity cyclotrons and describe the IsoDAR compact cyclotron design. Most of the focus will be given to the design of the central region of the cyclotron and the matching of the RFQ output beam to it. This will be done by discussing the $1 \mathrm{MeV} / \mathrm{amu}$ test cyclotron that was designed for RFQ-DIP and which is a prototype of the central region of the full IsoDAR cyclotron.

\subsubsection{Vortex Motion}

In high current cyclotrons, the combination of space charge and the external focusing forces of the cyclotron's azimuthally varying main magnetic field can lead to the formation of a stable, round (in the radial-longitudinal plane) bunch. For the case of the PSI Injector 2 cyclotron, this has been extensively studied and discussed (see for
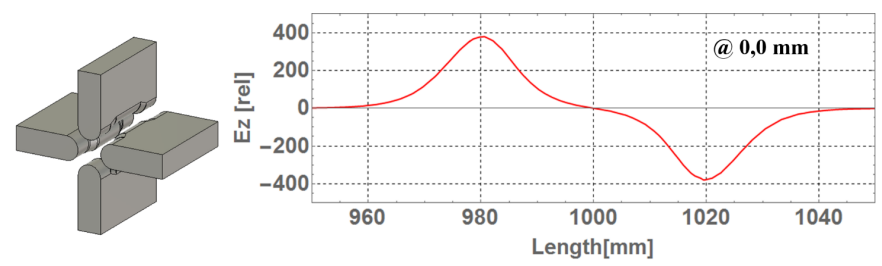

Figure 9: 3D CAD model of the trapezoidal re-buncher cell and associated field $E_{z}$ along the center axis. example [6, 7, 61]). The effect was dubbed vortex effect or vortex motion because the beam exhibits a vortex-like rotation in a local coordinate system (origin shifted to the bunch centroid position and local y-axis aligned with the bunch mean momentum). It has been shown that, to achieve stable vortex motion, the bunches have to be matched to the cyclotron through pre-bunching, focusing and carefully collimating the beam both before the cyclotron and inside, during the first few turns [7, 62, 63. In this context, the following critical items are addressed for IsoDAR and RFQ-DIP through the measures described:

1. Longitudinal bunch size and phase acceptance: The RFQ includes a trapezoidal re-bunching cell at the end (as described in the previous section) to optimize the number of particles within the phase acceptance window of the cyclotron (typically $\pm 10^{\circ}$ for isochronous machines) during the crossing of the first accelerating cavity.

2. Transverse beam spread: Firstly, the RFQ exit is brought very close to the entrance of the spiral inflector. Secondly, if necessary, there is room for a solenoid or quadrupole inside the cyclotron bore to provide additional focusing. Third, the spiral inflector includes a special "V-shape" for additional focusing in the cyclotron axial direction (see Section 2.3.5.

3. Scraping of halo particles. Particles that are outside of the phase acceptance of the cyclotron will inevitably become a nuisance, add to the beam halo and reduce the turn separation at the extraction point. Careful placement of collimators to scrape these particles early on is done in highly realistic simulations using the OPAL code 64.

These parameters will be further optimized during the final design process. 


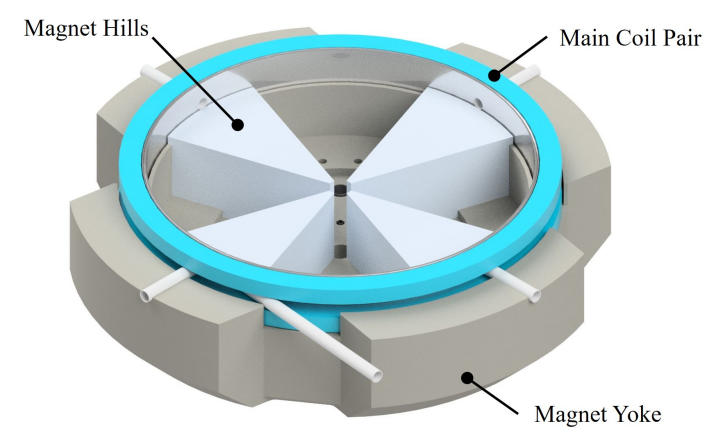

Figure 10: View of the bottom half of the full IsODAR cyclotron. The magnet yoke, magnet poles (hill region) and cyclotron main coil pair are indicated. Overall diameter: $6 \mathrm{~m}$.

\subsubsection{The full IsoDAR cyclotron}

The design of IsoDAR cyclotron and beam dynamics simulations starting during the third turn at $1.5 \mathrm{MeV} / \mathrm{amu}$ were presented in [65, 66] and [50, respectively. These reports were on the DAE $\delta$ ALUS Injector Cyclotron, which is identical to the IsoDAR cyclotron except for the working frequency which is $32.8 \mathrm{MHz}$ ( $4^{\text {th }}$ harmonic) for IsoDAR and $49.2 \mathrm{MHz}$ ( $6^{\text {th }}$ harmonic) for the DAE $\delta$ ALUS injector cyclotron. This cyclotron is a 4 -sector machine with a pole radius of about $220 \mathrm{~cm}$ and is able to accelerate $\mathrm{H}_{2}^{+}$ ions from the injection energy of $35 \mathrm{keV} / \mathrm{amu}$ up to 61.7 $\mathrm{MeV} / \mathrm{amu}$. The hill width varies from $25.5^{\circ}$ at the inner radii up to $36.5^{\circ}$ at outer radii. The hill gap is $100 \mathrm{~mm}$, while the valley gap is very large $(1800 \mathrm{~mm})$ to be able to contain the RF cavities. A half-section isometric view of the cyclotron can be seen in Figure 3 and a mid-plane cut in Figure 10. The main studies up to now were focused on the space charge effects along the acceleration path and on the extraction of the beam using an electrostatic deflector [50] using OPAL. In order to achieve sufficient turn separation at extraction, this cyclotron relies on the following:

1. High acceleration voltage. A key feature of this machine is to use RF cavities able to produce accelerating voltages as high as $240 \mathrm{kV}$ at the extraction region. This yields an inter-turn separation of about $13-14.8 \mathrm{~mm}$.

2. Precessional resonance crossing in the extraction region. A well-tuned fall-off of the magnetic field in the region of beam extraction leads to a crossing of the $\nu_{r}=1$ resonance (with $\nu_{r}$ the radial tune) a few centimeters before the extraction radius. This field configuration produces a precession of the accelerated orbit which increases the inter-turn separation to $20 \mathrm{~mm}$.

3. Vortex effect. This has been discussed in the previous section and collimator placements have been taken into account in [50]. The vortex effect does not increase the inter-turn separation, but prevents the beam from growing too large in the radial direction, which would lead to significant overlap even at high inter-turn separations.

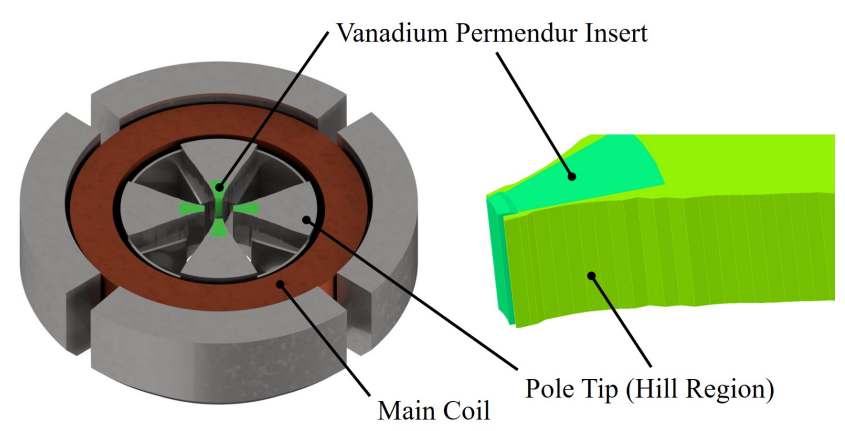

Figure 11: View of the bottom half of the new $1 \mathrm{MeV} / \mathrm{amu}$ cyclotron design with the Vanadium Permendur insert (green). Overall diameter: $1.5 \mathrm{~m}$

According to the simulations performed with OPAL [50], taking into account the space charge forces in a beam bunch with phase length of $10^{\circ} \mathrm{RF}$, the expected amount of beam loss on a $0.5 \mathrm{~mm}$ thick septum (the counter-part of the of the electrostatic deflector sitting between the last and second-to-last turn) should stay around $100 \mathrm{~W}$ for an extracted beam power of $600 \mathrm{~kW}$. For safety reasons, we also plan to use four graphite stripper foils placed around the last orbit trajectory, to remove the remaining beam halo and to reduce the beam power lost on the electrostatic deflector septum to virtually zero.

\subsubsection{The RFQ-DIP $1 \mathrm{MeV} /$ amu test cyclotron}

As mentioned before, to test the central region and the acceptance of the beam therein, a separate $1 \mathrm{MeV} / \mathrm{amu}$ test cyclotron was designed with a magnetic field and clearances for spiral inflector and collimators identical to the final IsoDAR machine (see Figure 11). Indeed, the previous design showed that the vertical focusing was not strong enough to focus the injected beam vertically. This was mainly due to the $10 \mathrm{~cm}$ vertical gap between the cyclotron magnet poles and due to the low flutter in the central region. To increase the flutter (and hence the vertical fo-

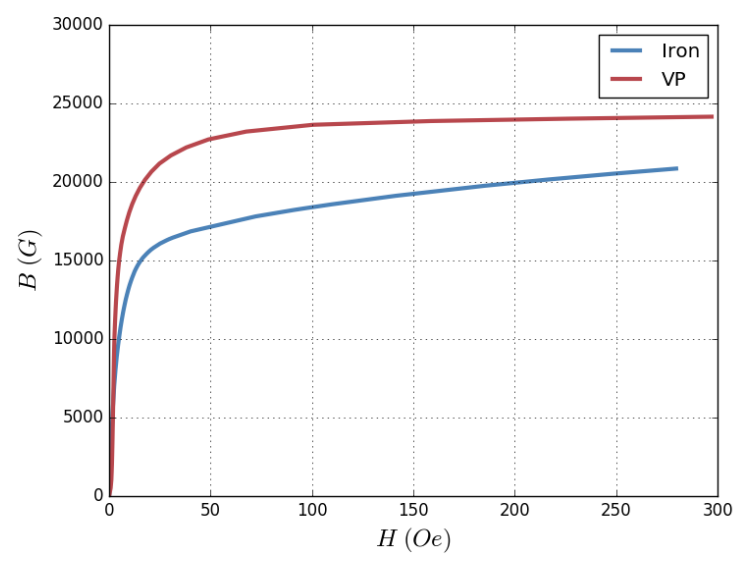

Figure 12: Magnetic permeability of soft iron and vanadium permendur (VP). 

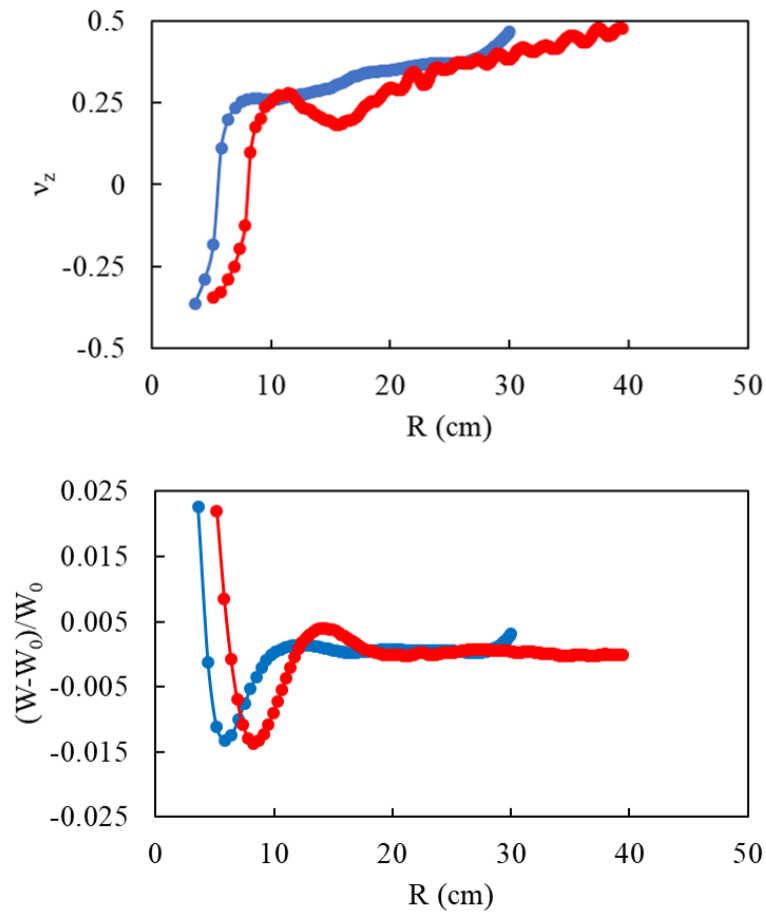

Figure 13: Upper: The $\nu_{z}$ values for the early model of IsoDAR in red and for the new $1 \mathrm{MeV} / \mathrm{amu}$ test machine in blue. Lower: Isochronism parameter $\left(W-W_{0}\right) / W_{0}$, for the early model of IsoDAR in red and for the new $1 \mathrm{MeV} / \mathrm{amu}$ test machine in blue

cusing), the pole gap was reduced and part of the iron of the hill in the central region was replaced with Vanadium Permendur (VP). Due to the higher magnetic permeability of the VP (see Figure 12, it is possible to achieve higher magnetic fields in the hill region, which leads to a higher flutter of the field along the orbit trajectories of the particles and consequently better vertical focusing.

The design parameters of this new test cyclotron are listed in Table 6. The main items to highlight are the VP inserts and the unusually large bore hole. The VP inserts start at a radius of $3.5 \mathrm{~cm}$ and end at $15 \mathrm{~cm}$. They are protected by soft iron that can be removed for the fine shimming. A closer view is presented in Figure 11, right. The bore hole has a diameter of $27 \mathrm{~cm}$ and allows insertion

Table 6: $1 \mathrm{MeV} / \mathrm{amu}$ test cyclotron design parameters.

\begin{tabular}{lrl}
\hline Parameter & Value & Unit \\
\hline Yoke outer diameter & 1.5 & $\mathrm{~m}$ \\
Bore diameter & 27 & $\mathrm{~cm}$ \\
Weight & 6.3 & tons \\
Pole angle & 23 to 46 & $\mathrm{deg}$ \\
Pole radius & 37 & $\mathrm{~cm}^{2}$ \\
Coil cross-section & $\times 18$ & $\mathrm{~cm}^{2}$ \\
Max. field on the mid-plane & 1.48 & $\mathrm{~T}$ \\
Min. field on the mid-plane & 0.5 & $\mathrm{~T}$ \\
Energy of last closed orbit & 1.4 & $\mathrm{MeV} / \mathrm{amu}$ \\
\hline
\end{tabular}

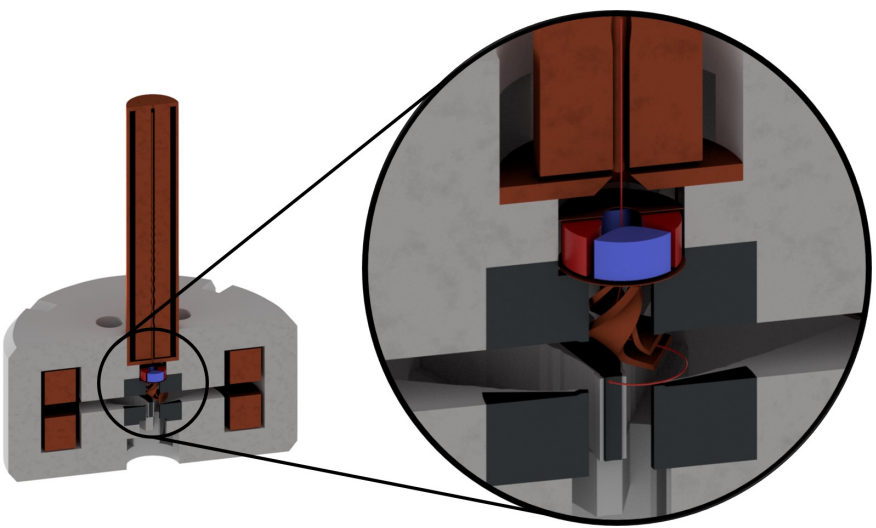

Figure 14: CAD rendering of the central region of the RFQ-DIP 1 $\mathrm{MeV} / \mathrm{amu}$ test cyclotron. The follwing items have been removed for visibility: Half the quadrupole entrance aperture, grounded housing of the spiral inflector, rf electrodes for acceleration in the cyclotron. An electrostatic quadrupole singlet (electrodes shown in red and blue) is placed between the RFQ and the spiral inflector for improved transmission and tunability.

of the RFQ into the cyclotron up to $20 \mathrm{~cm}$ from the midplane.

In Figure 13 (upper), the vertical tune $\nu_{z}$ is shown vs. radius for the IsoDAR cyclotron (red curve) and for the $1 \mathrm{MeV} / \mathrm{amu}$ test cyclotron. It is clearly visible that the value of $\nu_{z}$ has increased significantly at radii lower than $10 \mathrm{~cm}$. This is important to achieve a sufficient vertical focusing along the first orbit where space charge effects are still strong. In Figure 13 (lower), the isochronism parameter $\left(W-W_{0}\right) / W_{0}$ for the IsoDAR cyclotron and for the $1 \mathrm{MeV} / \mathrm{amu}$ machine are shown. Both machines exhibit good isochronism and match very well in the range between 18 to $28 \mathrm{~cm}$. This isochronism matching is important to guarantee that the beam accelerated in the $1 \mathrm{MeV} / \mathrm{amu}$ machine could seamlessly be accelerated further in the $60 \mathrm{MeV} / \mathrm{amu}$ IsoDAR machine.

As mentioned before, for the remainder of Section 2 , all considerations (transfer of the beam through the spiral inflector and RF electrode structure for the first few turns) will be with respect to the $1 \mathrm{MeV} / \mathrm{amu}$ RFQ-DIP test cyclotron. The assembly of test cyclotron, RFQ and the matching elements (electrostatic quadrupole singlet and spiral inflector) are shown in Figure 14. A single electrostatic quadrupole has been found to give a measure of tunability and increases the transmission of the beam through the spiral inflector from roughly $70 \%$ to $80 \%$.

\subsubsection{Design considerations for spiral inflectors}

Many of the spiral inflector design parameters depend on the beam and particle properties. Primarily, there are two free parameters: the applied electric field, and a tilt parameter, which is denoted by $k^{\prime}$. Electrode voltages and electrode gap width determine the electric bending radius of the spiral inflector, which corresponds exactly to the height of the device. $k^{\prime}$ describes an angle at which the exit of the electrodes is tilted. This parameter can be changed 


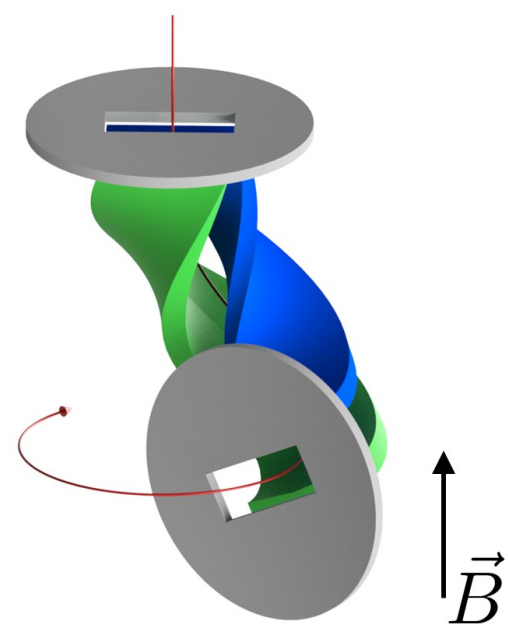

Figure 15: 3D view of the IsoDAR spiral inflector (anode: green, cathode: blue) and apertures (gray) with a reference $\mathrm{H}_{2}^{+}$trajectory (red).

to align center of curvature for the particle trajectories. In the analytical treatment of the spiral inflector, the magnetic field in the central region is taken to be constant in the axial direction. This assumption allows for an exact solution of a central ion trajectory through the spiral inflector which can be used to calculate the surfaces of the spiral electrodes [67].

For taller spiral inflectors, it may not be appropriate to assume the magnetic field is constant. The magnetic field of a cyclotron along the z-axis may vary significantly enough such that the beam does not follow the analytical central trajectory. Secondly, the fringing electric fields at the entrance and exit of the spiral inflector cause the beam to exit with an angle to the mid-plane of the cyclotron. To account for both the fringing electric field and a nonuniform magnetic field, numerical analyses and optimization routines have been developed in a python module to aid in the design process of spiral inflectors [68]. This code applies the analytical treatment of the spiral inflector and performs a full electric field calculation, which includes the fringe fields using a boundary elements method implemented in the python module BEMPP 69. A design particle is then tracked through the spiral inflector and the entrance and exit of the spiral inflector are modified to compensate for the effect of fringe fields.

\subsubsection{The IsoDAR/RFQ-DIP spiral inflector}

A preliminary design of the IsoDAR spiral inflector, including entrance and exit apertures, is presented in Figure 15. The geometry of the spiral inflector was generated using the newly developed code presented in Section 2.3.4 using a numerical analysis to correct for fringe field effects and a non-uniform magnetic field. The aspect ratio between the cross-sectional width and length of the spiral electrodes was set to be 2.5. The opening of the grounded apertures were designed to match the shape of the entrance and exit of the spiral inflector to shield the

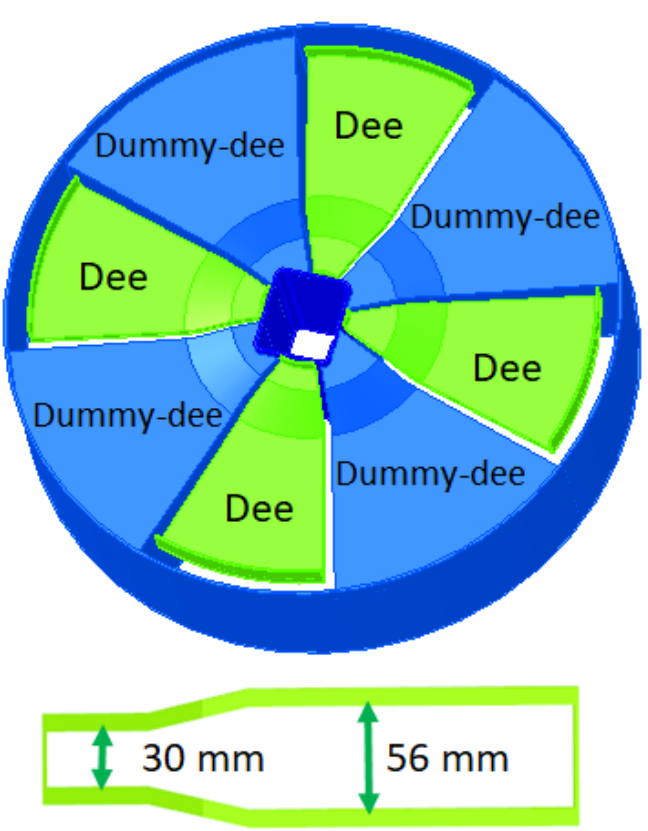

Figure 16: $3 \mathrm{D}$ view of the IsoDAR central region. The dummy-dees are in blue while the dees are in green.

incoming particles from the strong fringing electric fields and to collimate the beam. The top aperture is placed 5 $\mathrm{mm}$ from the entrance of the spiral inflector and the bottom aperture is placed $10 \mathrm{~mm}$ from the exit, each with a thickness of $4 \mathrm{~mm}$.

The high beam current that is being delivered to the cyclotron necessitates a large initial gap distance of $1.8 \mathrm{~cm}$ to minimize the loss of particles during injection. Additionally, the voltage assigned to the electrodes was chosen to be $\pm 12 \mathrm{kV}$. The IsoDAR spiral inflector is taller than a typical spiral inflector because of the gap distance since the height is inversely proportional to the strength of the electric field between the electrodes.

\subsubsection{The IsoDAR Central Region}

The final element of the RFQ-DIP test cyclotron is the RF system consisting of dees and dummy-dees. A rendering of the preliminary design is shown in Figure 16. After leaving the spiral inflector, the $\mathrm{H}_{2}^{+}$ions are injected into the cyclotron central region at $70 \mathrm{keV}$ energy and accelerated up to the $1 \mathrm{MeV} / \mathrm{amu}$. The accelerating structure is

Table 7: Spiral inflector design parameters.

\begin{tabular}{lrl}
\hline Parameter & Value & Unit \\
\hline Electrode voltages & \pm 12 & $\mathrm{kV}$ \\
Input energy & 70 & $\mathrm{keV}$ \\
Electrode width & 1.0 & $\mathrm{~cm}$ \\
Gap distance & 1.8 & $\mathrm{~cm}$ \\
Aspect ratio & 2.5 & \\
Tilt angle & 27 & $\mathrm{deg}$ \\
\hline
\end{tabular}



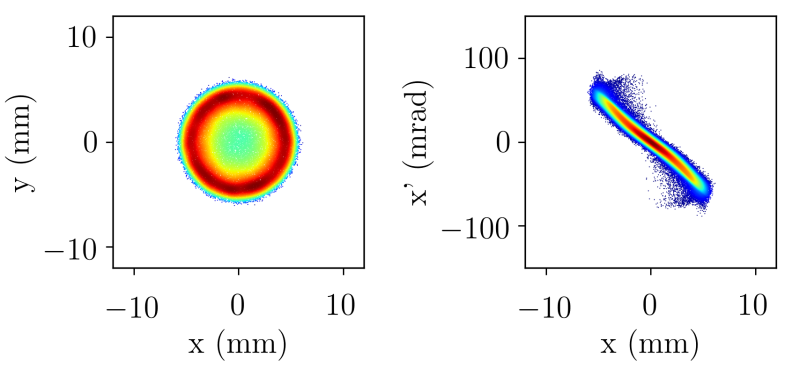

Figure 17: Beam cross-section (left) and $\mathrm{xx}^{\prime}$ phase space (right) for $8 \mathrm{~mA}$ of $\mathrm{H}_{2}^{+}$(transported together with $2 \mathrm{~mA}$ of protons) at the entrance flange of the RFQ. The beam is converging so that the transverse focus lies well within the RFQ's radial matching section. Corresponding beam parameters are listed in Table 8

composed of four electrodes, the so-called dees, driven by a $\mathrm{RF}$ voltage with a maximum of $70 \mathrm{kV}$ and by as many electrodes at ground, usually named dummy-dees.

The RF resonance frequency of the cavities is $32.8 \mathrm{MHz}$ and the accelerating harmonic is the $4^{\text {th }}$, four times the revolution frequency of the beam in the magnetic field of the IsoDAR cyclotron. The vertical aperture of the electrodes has two different values, $30 \mathrm{~mm}$ in the first turn and $56 \mathrm{~mm}$ after. The choice of smallest vertical gap at the inner cyclotron radii allows to reduce the transit time factor effects on the beam dynamics in the first turn.

Finally, the angular width of the dees is 36 degrees and the gap between each dee and dummy-dee is $12 \mathrm{~mm}$.

While the preliminary results of the RF system are promising (as reported in Section 3.3), further optimization is necessary, and a detailed design study performed by the AIMA company in France is currently on the way.

\section{Simulations}

In the following section, the latest simulation results of the RFQ-DIP design will be described briefly. Simulations showing the feasibility of the IsoDAR main cyclotron design by accelerating high intensity beams from later turns have been reported in [50] (starting at $1.5 \mathrm{MeV} / \mathrm{amu}$ ) and 51] (starting at $193 \mathrm{keV} / \mathrm{amu}$ ) and are not subject of this manuscript. A detailed report on the full start-to-end simulations of RFQ-DIP and IsoDAR is forthcoming.

\subsection{Initial conditions from ion source}

The initial conditions for the RFQ simulations are obtained from simulations of the ion source plasma extraction using IBSIMU, as was described in Section 2.1. We consider two cases: the nominal case of $10 \mathrm{~mA}$ total extracted beam current and the exaggerated case of $20 \mathrm{~mA}$ total extracted beam current (both with $80 \% \mathrm{H}_{2}^{+}, 20 \%$ protons). The beam parameters at the entrance flange of the RFQ (rightmost electrode in Figure 5) are listed in Table 8 and a representative phase space is shown in Figure 17.
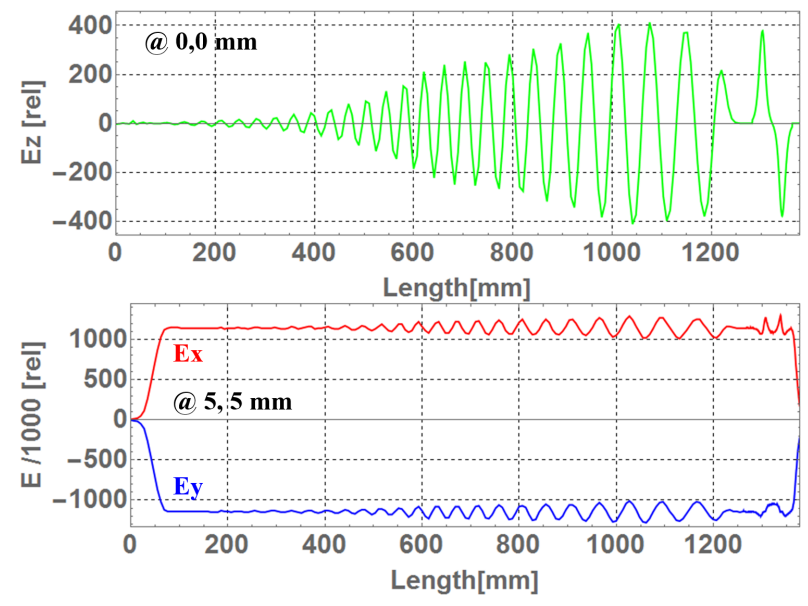

Figure 18: The field distribution along beam axis with re-buncher.

\section{2. $R F Q$ simulations}

As a part of the RFQ-DIP and IsoDAR systems, the RFQ accelerator provides acceleration of the ion beam generated from the ion source and highly efficient bunching as well as beam separation by different mass-to-charge ratios ( 1 for the proton beam and 2 for the $\mathrm{H}_{2}^{+}$ion beam). In this section, we described the beam dynamics in the RFQ accelerator using the particle distributions from the previously reported IBSIMU results. The electric field distributions along the beam axis and $5 \mathrm{~mm}$ offset are presented in Figure 18. These include the re-buncher fields in the transition cell of the designed IsoDAR RFQ, to obtain additional longitudinal focusing.

As an operational scenario, studies with $10 \mathrm{~mA}$ and $20 \mathrm{~mA}$ of beam intensity have been performed. The initial beam distributions of the two cases at the entrance of the IsoDAR RFQ are presented in Figure 19. Note that the upper row in Figure 19 corresponds directly to the distribution shown in Figure 17. The generated particles from the ion source, including additional, unwanted species, such as protons and $\mathrm{H}_{3}^{+}$, need to be separated and selected by the RFQ. When particles with the wrong mass-to-charge ratio are injected into the RFQ, they will not get accelerated, due to the improper RF frequency fields. Using the injected beam distributions from the ion

Table 8: Initial beam parameters for RFQ simulations obtained from IBSIMU calculations. (only the horizontal direction is shown, the vertical direction is similar).

\begin{tabular}{lcccc}
\hline & \multicolumn{2}{c}{$10 \mathrm{~mA}$} & \multicolumn{2}{c}{$20 \mathrm{~mA}$} \\
& protons & $\mathrm{H}_{2}^{+}$ & protons & $\mathrm{H}_{2}^{+}$ \\
\hline Fraction (\%) & 20 & 80 & 20 & 80 \\
Current (mA) & 2 & 8 & 4 & 16 \\
Energy (keV) & 15 & 15 & 15 & 15 \\
$\varnothing_{2 \mathrm{rms}}(\mathrm{mm})$ & 11.8 & 11.8 & 14.4 & 14.4 \\
$\epsilon_{\mathrm{x}, \mathrm{n}, 4 \mathrm{rms}}(\pi$-mm-mrad) & 0.60 & 0.43 & 0.91 & 0.65 \\
4rms includes $(\%)$ & 90.7 & 90.7 & 88.4 & 88.3 \\
\hline
\end{tabular}



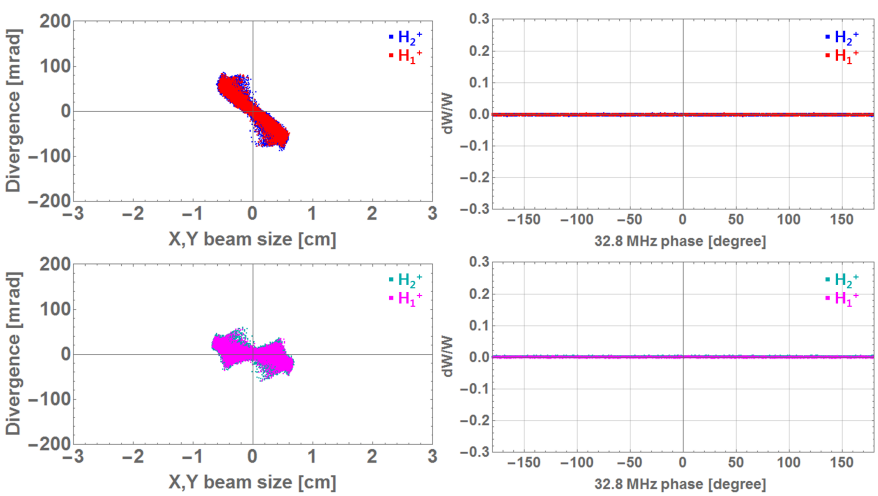

Figure 19: The transverse and longitudinal beam distributions at the entrance of the IsoDAR RFQ in cases of $10 \mathrm{~mA}$ (top) and $20 \mathrm{~mA}$ (bottom)
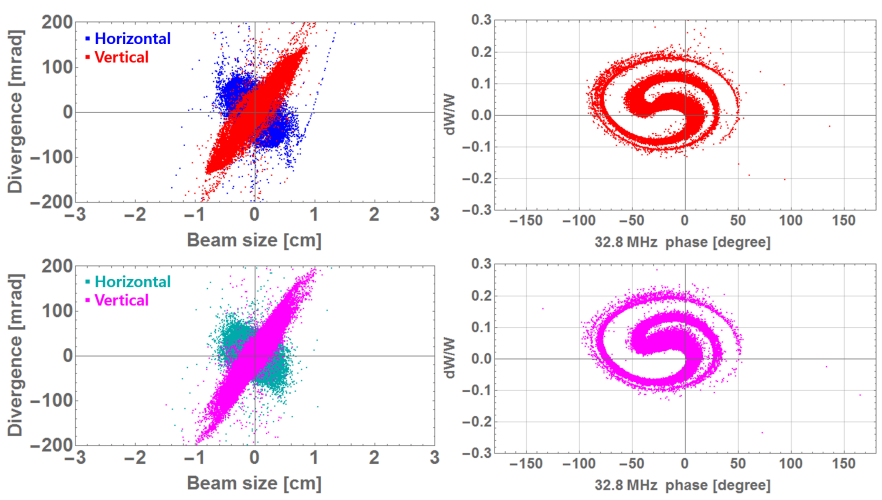

Figure 20: The transverse and longitudinal beam distributions at the exit of the IsoDAR RFQ for the two cases of $10 \mathrm{~mA}$ (top) and $20 \mathrm{~mA}$ (bottom)

source, the results of the RFQ simulations for both cases have transmission efficiencies above $97 \%$ and 8.186 and $6.908 \mathrm{keV} / \mathrm{u}$-ns of longitudinal emittance, respectively. 3D tracking was done using the TRACK code. The RMS envelopes of transverse beam size for the two cases of $10 \mathrm{~mA}$ and $20 \mathrm{~mA}$ of beam intensity are presented in Figure 21. They are smaller than $2.96 \mathrm{~mm}$ for $10 \mathrm{~mA}$ and $3.88 \mathrm{~mm}$ for $20 \mathrm{~mA}$, respectively. The beam distributions at the end of the RFQ are shown in Figure 20. As expected, the transverse emittances increase with beam intensity, due to space charge effects. The detailed simulation results for the two cases are summarized in Table 9

Table 9: Important resulting parameters of the RFQ simulations for the current RFQ-DIP/IsoDAR design ( $\mathrm{r}=\mathrm{rms}$, $\mathrm{n}=$ normalized).

\begin{tabular}{llll}
\hline Elements & $10 \mathrm{~mA}$ & $20 \mathrm{~mA}$ & units \\
\hline Transmission rate & 98.48 & 97.12 & $\%$ \\
Input tr. emit $(\mathrm{r}, \mathrm{n})$ & 0.106 & 0.304 & $\mathrm{~mm}-\mathrm{mrad}$ \\
Output emit x $(\mathrm{r}, \mathrm{n})$ & 0.306 & 0.373 & $\mathrm{~mm}-\mathrm{mrad}$ \\
Output emit y $(\mathrm{r}, \mathrm{n})$ & 0.329 & 0.393 & $\mathrm{~mm}-\mathrm{mrad}$ \\
Output emit z $(\mathrm{r})$ & 8.186 & 6.908 & $\mathrm{keV} / \mathrm{u}-\mathrm{ns}$ \\
\hline
\end{tabular}
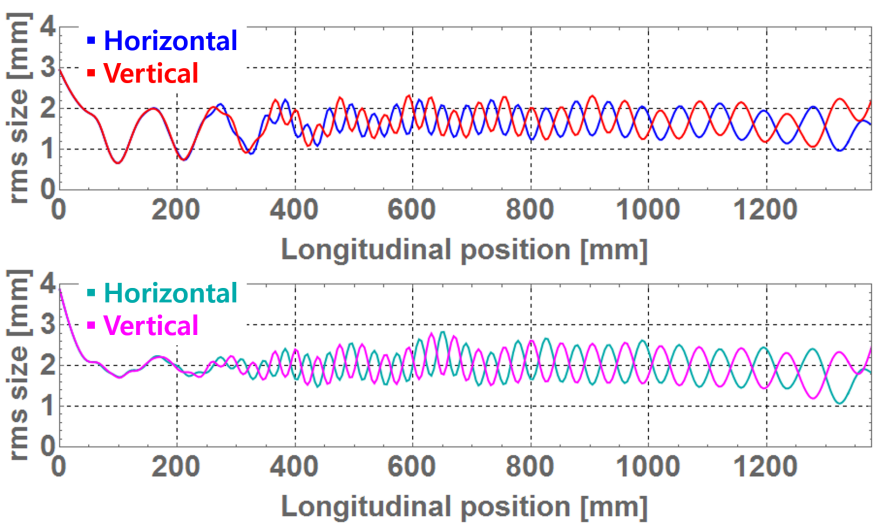

Figure 21: The RMS beam size along the RFQ cavity for the two cases of $10 \mathrm{~mA}$ (top) and $20 \mathrm{~mA}$ (bottom).

\subsection{Spiral inflector and central region simulations}

The simulation studies of the IsoDAR spiral inflector and central region reported here, were performed in two steps:

1. Preliminary simulation study in collaboration with the company IBA in Belgium.

2. Detailed 3D simulation study including space charge (ongoing effort at MIT and the company AIMA in France).

Preliminary simulations. The results of the preliminary simulations can be found here 70 and are briefly summarized below. By using an iterative approach, the spiral inflector and central region have been designed and simulated to guarantee good injection and acceleration of the reference orbit in the test cyclotron center. The motion of a single particle, starting from a position along the cyclotron vertical axis (100 $\mathrm{mm}$ from the median plane, 20 $\mathrm{mm}$ from the spiral inflector entrance) was tracked. The initial RF phase of the reference orbit was selected with the aim to optimize the vertical excursion in the cyclotron center, the horizontal orbit centering and the energy gain per turn. The designed spiral inflector and central region provide a good injection and acceleration of the reference orbit in the test cyclotron. To test transmission, 5000 particles with the same distribution in the both $\mathrm{x}-\mathrm{x}^{\prime}$ and $\mathrm{y}-\mathrm{y}^{\prime}$ phase planes were then simulated. A normalized emittance of $1 \pi \cdot \mathrm{mm} \cdot \mathrm{mrad}$ was used, a value that is about $20 \%$ larger than the expected beam emittance delivered by the RFQ. A round, uniform beam with $10 \mathrm{~mm}$ diameter has been considered. In addition an energy spread equal to $\pm 2 \%$ has been taken into account. The results of the simulations show that losses are localized in the cyclotron center, in particular at the inflector exit and in the first half turn, since the vertical aperture of the housing hole and electrodes is smaller than beam vertical envelope. This is due to the growth of beam emittance through the spiral inflector and the transverse defocusing of the beam at the inflector exit. The space charge effects play an important role in the beam growth and transverse defocusing 
determining an increase of the losses. In addition, turn separation has been observed to be too small, due to the overlap between the tails of the beam distribution of adjacent turns. Further optimization and inclusion of the actual RFQ output beam distribution was deemed necessary.

Detailed simulations. the first step in the detailed simulation study was to generate a spiral inflector using the methods described in Section 2.3.4. The electrostatic quadrupole singlet was added and the fields were calculated in COMSOL Multiphysics. The RFQ output particles presented in the previous subsection were then tracked, including space charge, for $10 \mathrm{~mA}$ and $20 \mathrm{~mA}$ of beam. The result of the $20 \mathrm{~mA}$ case is shown in Figure 22. The beam was well-centered on the mid-plane with $12.3 \mathrm{kV}$ applied to the spiral electrodes and has a maximum vertical extent of $35 \mathrm{~mm}$. The transmission in both cases was around $80 \%$ when using $11.5 \mathrm{kV}$ for the quadrupole. Most of the losses occur towards the end of the inflector due to the initial energy spread of the beam. Further reduction of the beam energy spread can be achieved with a vertical aperture close to the exit of the inflector, albeit at the expense of higher losses. These particles are now being used in the optimization of the central region by AIMA. This process includes shimming the magnetic field to reduce first harmonic perturbations, shaping the dees and placing pillars (collimators) along the beam path in the central region. This removes the beam halo radially in order to achieve well separated turns. The use of pillars will also allow to increase the electrode vertical aperture to reduce the vertical losses and the transit time factor will be mitigated by the smaller radial aperture for the beam.

As mentioned above, this simulation effort is ongoing and another paper on the full start-to-end simulations of the RFQ-DIP and IsoDAR projects is forthcoming.

\section{Summary and Outlook}

The prospect of being able to reliably create and deliver cw proton beam currents of $10 \mathrm{~mA}$ and more at energies upwards of $60 \mathrm{MeV}$ is very exciting for the fields of particle physics (e.g. neutrino experiments [8, 9, 10, 11]), medical isotope production [16, 17, and materials research (see for example [7]). A system that can perform at those levels was described in this paper. It consists of a high intensity $\mathrm{H}_{2}^{+}$ion source, RFQ injection system, and compact cyclotron for acceleration. As was discussed here, the main challenges are controlling space charge and achieving high injection efficiency into the cyclotron. We have shown preliminary designs and simulations showing that both are possible using $\mathrm{H}_{2}^{+}$as the primary ion, an RFQ to aggressively pre-bunch the beam before injecting it through a spiral inflector, and by carefully designing a central region that facilitates vortex motion and uses collimators to clean up beam halo. The beam was matched to the central region of the cyclotron and acceleration for 4 turns

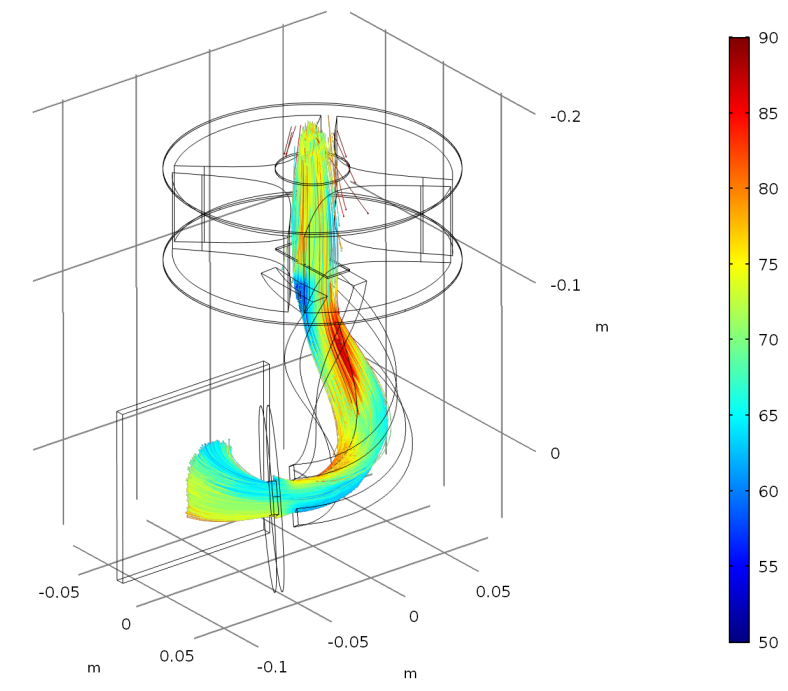

Figure 22: The RFQ output particle distribution tracked through the electrostatic quadrupole singlet and the spiral inflector, using COMSOL Multiphysics. the particles are terminated on a beam stop immediately after exiting the inflector. Colorbar: particle kinetic energy (keV).

showed good turn separation as well as the onset of vortex motion, which has a stabilizing effect on the beam. The next steps on the path to a widely usable high intensity proton driver, will be the successful completion of the RFQ-DIP, demonstrating RFQ direct injection into a $1 \mathrm{MeV} / \mathrm{amu}$ test cyclotron, followed by the full IsoDAR machine, which will be able to deliver $600 \mathrm{~kW}$ of beam power $(10 \mathrm{~mA} \mathrm{cw}$ protons at $60 \mathrm{MeV}$ after a stripper foil). As was described in Section 2 the RFQ - Direct Injection Project is well underway and we expect completion of the test bench and first results by the end of 2019. A proposal for the full IsoDAR system will be submitted by the end of 2018 .

Once the high intensity $\mathrm{H}_{2}^{+}$beam has been extracted from the compact cyclotron, one can envision to inject it into another cyclotron to boost the beam energy even higher. This process is described in detail elsewhere 10 , 11 for the case of (DAE $\delta$ ALUS), another neutrino experiment searching for cp-violation. Here a separated sector superconducting cyclotron is used to accelerate $\mathrm{H}_{2}^{+}$from $60 \mathrm{MeV} / \mathrm{amu}$ to $800 \mathrm{MeV} / \mathrm{amu}$. However, one does not have to stop at $800 \mathrm{MeV} / \mathrm{amu}$, and with the technology developed here, multi-megawatt compact systems using two chained cyclotrons are now within reach for energy research (needs described for example in [12, 13, 14, 15]).

\section{Acknowledgements}

MIST-1 is supported by NSF grant \#PHY-1505858 and funding from the Bose Foundation. The RFQ-DIP project is supported by NSF grant \#PHY-1626069 and the Heising-Simons Foundation. The authors are very thankful for the support of the MIT Central Machine shop, the 
MIT Plasma Science and Fusion Center (PSFC), and the University of Huddersfield for support with machining, lab space and utilities, and equipment, respectively. The corresponding author would also like to thank the American Physical Society and the Indo-U.S. Science and Technology Forum for awarding him a travel grant through the Ph.D. student and Postdoc Visitation Program to go to Kolkata and collaborate on RFQ design with the RIB group at VECC.

\section{References}

\section{References}

[1] T. Mason, D. Abernathy, I. Anderson, J. Ankner, T. Egami, G. Ehlers, A. Ekkebus, G. Granroth, M. Hagen, K. Herwig, et al., The Spallation Neutron Source in Oak Ridge: A powerful tool for materials research, Physica B: Condensed Matter 385 (2006) 955-960.

[2] F. Maekawa, M. Harada, K. Oikawa, M. Teshigawara, T. Kai, S. ichiro Meigo, M. Ooi, S. Sakamoto, H. Takada, M. Futakawa, T. Kato, Y. Ikeda, N. Watanabe, T. Kamiyama, S. Torii, R. Kajimoto, M. Nakamura, First neutron production utilizing JPARC pulsed spallation neutron source JSNS and neutronic performance demonstrated, Nuclear Instruments and Methods in Physics Research Section A: Accelerators, Spectrometers, Detectors and Associated Equipment 620 (2) (2010) 159 - 165. doi:https://doi.org/10.1016/j.nima.2010.04.020

I URL http://www.sciencedirect.com/science/article/pii/ S0168900210008223

[3] W. Fischer, SINQ The spallation neutron source, a new research facility at PSI, in: AIP Conference Proceedings, Vol. 392, AIP, 1997, pp. 1119-1122.

[4] D. Akimov, et al., Observation of Coherent Elastic NeutrinoNucleus Scattering, Science 357 (6356) (2017) 1123-1126. arXiv: 1708.01294 doi:10.1126/science.aao0990

[5] M. Seidel, S. Adam, A. Adelmann, C. Baumgarten, Y. Bi, R. Doelling, H. Fitze, A. Fuchs, M. Humbel, J. Grillenberger, et al., Production of a 1.3 MW proton beam at PSI in: Proceedings of the $1^{\text {st }}$ International Particle Accelerator Conference (2010), 2010, p. 1309.

II URL https://accelconf.web.cern.ch/AccelConf/IPAC10/ papers/tuyra03.pdf

[6] T. Stammbach, S. Adam, H. Fitze, W. Joho, M. Märki, M. Olivo, L. Rezzonico, P. Sigg, U. Schryber, The feasibility of high power cyclotrons Nuclear Instruments and Methods in Physics Research Section B: Beam Interactions with Materials and Atoms 113 (1) (1996) 1-7, accelerators in Applied Research and Technology. doi:https://doi.org/10. 1016/0168-583X (95) 01377-6

URL http://www.sciencedirect.com/science/article/pii/ $0168583 \times 95013776$

[7] J. Yang, A. Adelmann, M. Humbel, M. Seidel, T. Zhang, et al., Beam dynamics in high intensity cyclotrons including neighboring bunch effects: Model, implementation, and application, Physical Review Special Topics-Accelerators and Beams 13 (6) (2010) 064201.

[8] A. Bungau, et al., Proposal for an Electron Antineutrino Disappearance Search Using High-Rate ${ }^{8} \mathrm{Li}$ Production and Decay, Phys. Rev. Lett. 109 (2012) 141802. arXiv:1205.4419, doi:10.1103/PhysRevLett.109.141802

[9] M. Abs, et al., IsoDAR@KamLAND: A Conceptual Design Report for the Technical Facility arXiv: 1511.05130

[10] M. Abs, et al., Multimegawatt DAE $\delta$ ALUS Cyclotrons for Neutrino PhysicsarXiv: 1207.4895

[11] C. Aberle, A. Adelmann, J. Alonso, W. Barletta, R. Barlow, L. Bartoszek, A. Bungau, A. Calanna, D. Campo, L. Calabretta, et al., Whitepaper on the DAE $\delta$ ALUS programarXiv: 1307.2949
[12] Y. Ishi, M. Inoue, Y. Kuriyama, Y. Mori, T. Uesugi, J. Lagrange, T. Planche, M. Takashima, E. Yamakawa, H. Imazu, et al., Present status and future of FFAGs at KURRI and the first ADSR experiment, in: Proceedings of IPAC10, Kyoto, 2010, p. 1323.

[13] C. Rubbia, C. Roche, J. A. Rubio, F. Carminati, Y. Kadi, P. Mandrillon, J. P. C. Revol, S. Buono, R. Klapisch, N. Fiétier, et al., Conceptual design of a fast neutron operated high power energy amplifier, Tech. rep. (1995).

[14] J. Biarrotte, D. Uriot, F. Bouly, D. Vandeplassche, J. Carneiro, Design of the MYRRHA 17-600 MeV superconducting linac, in: Proceedings of SRF13, 2013.

[15] P. Lisowski, The accelerator production of tritium, in: Proceedings of PAC97, Vol. 97, 1997, p. 3780.

[16] P. Schmor, Review of cyclotrons used in the production of radioisotopes for biomedical applications, in: Proceedings of CYCLOTRONS, 2010, pp. 419-424.

[17] J. R. Alonso, Relevance of IsoDAR and DAE $\delta$ ALUS to Medical Radioisotope Production, ArXiv e-printsarXiv:1209.4925.

[18] J. R. Alonso, K. Nakamura, IsoDAR@KamLAND:A Conceptual Design Report for the Conventional FacilitiesarXiv:1710. 09325 .

[19] F. An, et al., Neutrino Physics with JUNO, J. Phys. G43 (3) (2016) 030401. arXiv:1507.05613, doi:10.1088/0954-3899/ 43/3/030401

[20] A. Aguilar-Arevalo, et al., Evidence for neutrino oscillations from the observation of anti-neutrino(electron) appearance in a anti-neutrino(muon) beam, Phys. Rev. D64 (2001) 112007. arXiv:hep-ex/0104049 doi:10.1103/PhysRevD.64.112007

[21] A. A. Aguilar-Arevalo, et al., A Search for Electron Antineutrino Appearance at the $\Delta m^{2} \sim 1 \mathrm{eV}^{2}$ Scale, Phys. Rev. Lett. 103 (2009) 111801. arXiv:0904.1958 doi:10.1103/ PhysRevLett.103.111801

[22] A. A. Aguilar-Arevalo, et al., Improved Search for $\bar{\nu}_{\mu} \rightarrow \bar{\nu}_{e}$ Oscillations in the MiniBooNE Experiment, Phys. Rev. Lett. 110 (2013) 161801. arXiv:1303.2588 doi:10.1103/PhysRevLett. 110.161801

[23] G. Mention, M. Fechner, T. Lasserre, T. A. Mueller, D. Lhuillier, M. Cribier, A. Letourneau, The Reactor Antineutrino Anomaly, Phys. Rev. D83 (2011) 073006. arXiv:1101.2755, doi:10.1103/PhysRevD.83.073006

[24] C. Zhang, X. Qian, P. Vogel, Reactor Antineutrino Anomaly with known $\theta_{13}$, Phys. Rev. D87 (7) (2013) 073018. arXiv: 1303.0900 doi:10.1103/PhysRevD.87.073018

[25] J. N. Abdurashitov, et al., Measurement of the solar neutrino capture rate with gallium metal. III: Results for the 2002-2007 data-taking period, Phys. Rev. C80 (2009) 015807. arXiv: 0901. 2200 doi:10.1103/PhysRevC.80.015807

[26] F. Kaether, W. Hampel, G. Heusser, J. Kiko, T. Kirsten, Reanalysis of the GALLEX solar neutrino flux and source experiments, Phys. Lett. B685 (2010) 47-54. arXiv:1001.2731. doi:10.1016/j.physletb.2010.01.030

[27] B. Armbruster, et al., Upper limits for neutrino oscillations muon-anti-neutrino $\rightarrow$ electron-anti-neutrino from muon decay at rest, Phys. Rev. D65 (2002) 112001. arXiv:hep-ex/0203021. doi:10.1103/PhysRevD.65.112001

[28] J. M. Conrad, M. H. Shaevitz, Limits on Electron Neutrino Disappearance from the KARMEN and LSND $\nu_{e}$ - Carbon Cross Section Data, Phys. Rev. D85 (2012) 013017. arXiv:1106.5552, doi:10.1103/PhysRevD.85.013017

[29] P. Adamson, et al., First Measurement of $\nu_{\mu}$ and $\nu_{e}$ Events in an Off-Axis Horn-Focused Neutrino Beam, Phys. Rev. Lett. 102 (2009) 211801. arXiv:0809.2447 doi:10.1103/PhysRevLett. 102.211801

[30] G. Cheng, et al., Dual baseline search for muon antineutrino disappearance at $0.1 \mathrm{eV}^{2}<\Delta m^{2}<100 \mathrm{eV}^{2}$, Phys. Rev. D86 (2012) 052009. arXiv:1208.0322 doi:10.1103/PhysRevD.86. 052009

[31] K. B. M. Mahn, et al., Dual baseline search for muon neutrino disappearance at $0.5 \mathrm{eV}^{2}<\Delta m^{2}<40 \mathrm{eV}^{2}$, Phys. Rev. D85 (2012) 032007. arXiv:1106.5685 doi:10.1103/PhysRevD.85. 
032007

[32] P. Astier, et al., Search for $\mathrm{nu}(\mathrm{mu}) \rightarrow \mathrm{nu}(\mathrm{e})$ oscillations in the NOMAD experiment, Phys. Lett. B570 (2003) 19-31. arXiv: hep-ex/0306037, doi:10.1016/j.physletb.2003.07.029

[33] I. E. Stockdale, et al., Limits on Muon Neutrino Oscillations in the Mass Range $55 \mathrm{eV}^{2}<\Delta m^{2}<800 \mathrm{eV}^{2}$, Phys. Rev. Lett. 52 (1984) 1384. doi:10.1103/PhysRevLett.52.1384

[34] F. Dydak, et al., A Search for Muon-neutrino Oscillations in the $\Delta m^{2}$ Range $0.3 \mathrm{eV}^{2}$ to $90 \mathrm{eV}^{2}$, Phys. Lett. 134B (1984) 281. doi:10.1016/0370-2693(84)90688-9

[35] G. H. Collin, C. A. Argüelles, J. M. Conrad, M. H. Shaevitz, Sterile Neutrino Fits to Short Baseline Data, Nucl. Phys. B908 (2016) 354-365. arXiv:1602.00671 doi:10.1016/j. nuclphysb.2016.02.024

[36] J. M. Conrad, M. H. Shaevitz, Sterile Neutrinos: An Introduction to Experiment: $\operatorname{arXiv:1609.07803}$

[37] J. Ashenfelter, et al., The PROSPECT Physics Program, J. Phys. G43 (11) (2016) 113001. arXiv:1512.02202 doi:10. 1088/0954-3899/43/11/113001

[38] M. Wurm, SOX: Short Distance Neutrino Oscillations with Borexino in: 25th International Workshop on Weak Interactions and Neutrinos (WIN2015), 2015.

I. URL https://www.mpi-hd.mpg.de/WIN2015/pages/program. html

[39] G. H. Collin, C. A. Argüelles, J. M. Conrad, M. H. Shaevitz, First Constraints on the Complete Neutrino Mixing Matrix with a Sterile Neutrino, Phys. Rev. Lett. 117 (22) (2016) 221801. arXiv:1607.00011, doi:10.1103/PhysRevLett.117.221801

[40] Y. Abe, et al., Measurement of 13 in Double Chooz using neutron captures on hydrogen with novel background rejection techniques, JHEP 01 (2016) 163. arXiv:1510.08937 doi: 10.1007/JHEP01(2016)163

[41] J. H. Choi, et al., Observation of Energy and Baseline Dependent Reactor Antineutrino Disappearance in the RENO Experiment, Phys. Rev. Lett. 116 (21) (2016) 211801. arXiv: 1511.05849 doi:10.1103/PhysRevLett.116.211801

[42] F. P. An, et al., Measurement of the Reactor Antineutrino Flux and Spectrum at Daya Bay, Phys. Rev. Lett. 116 (6) (2016) 061801, [Erratum: Phys. Rev. Lett.118,no.9,099902(2017)]. arXiv:1508.04233, doi:10.1103/ PhysRevLett.116.061801,10.1103/PhysRevLett.118.099902

[43] Y. Declais, et al., Search for neutrino oscillations at 15meters, 40-meters, and 95-meters from a nuclear power reactor at Bugey, Nucl. Phys. B434 (1995) 503-534. doi:10.1016/ 0550-3213(94)00513-E

[44] F. P. An, et al., Evolution of the Reactor Antineutrino Flux and Spectrum at Daya Bay, Phys. Rev. Lett. 118 (25) (2017) 251801. arXiv:1704.01082, doi:10.1103/PhysRevLett.118.251801

[45] M. Dentler, . Hernndez-Cabezudo, J. Kopp, M. Maltoni, T. Schwetz, Sterile neutrinos or flux uncertainties? Status of the reactor anti-neutrino anomaly, JHEP 11 (2017) 099. arXiv: 1709.04294, doi:10.1007/JHEP11(2017)099

[46] A. C. Hayes, G. Jungman, E. A. McCutchan, A. A. Sonzogni, G. T. Garvey, X. Wang, Analysis of the Daya Bay Reactor Antineutrino Flux Changes with Fuel Burnup, Phys. Rev. Lett. 120 (2) (2018) 022503. arXiv:1707.07728, doi: 10.1103/PhysRevLett.120.022503

[47] C. Giunti, X. P. Ji, M. Laveder, Y. F. Li, B. R. Littlejohn, Reactor Fuel Fraction Information on the Antineutrino Anomaly, JHEP 10 (2017) 143. arXiv:1708.01133, doi:10. 1007/JHEP10 (2017) 143

[48] J. M. Conrad, M. H. Shaevitz, I. Shimizu, J. Spitz, M. Toups, L. Winslow, Precision $\bar{\nu}_{e}$-electron scattering measurements with IsoDAR to search for new physics, Phys. Rev. D89 (7) (2014) 072010. arXiv:1307.5081 doi:10.1103/PhysRevD.89.072010

[49] G. P. Zeller, et al., A Precise determination of electroweak parameters in neutrino nucleon scattering, Phys. Rev. Lett. 88 (2002) 091802, [Erratum: Phys. Rev. Lett.90,239902(2003)]. arXiv:hep-ex/0110059 doi:10.1103/PhysRevLett.88.091802

[50] J. Yang, A. Adelmann, W. Barletta, L. Calabretta, A. Calanna, D. Campo, J. Conrad, Beam dynamics simulation for the high intensity DAE $\delta$ ALUS cyclotrons, Nuclear Instruments and Methods in Physics Research Section A: Accelerators, Spectrometers, Detectors and Associated Equipment 704 (2013) 84 - 91. doi:https://doi.org/10.1016/j.nima.2012.12.050

[51] J. Jonnerby, Central Region Design of a Compact High Intensity Cyclotron, Master's thesis, ETH Zürich, Switzerland (2016)

URL ETH-Accel-Lecture-1/projectscompleted/phys/JJMSc.pdf

[52] K. Ehlers, K. Leung, High-concentration H2+ or D2+ ion source, Review of Scientific Instruments 54 (6) (1983) 677-680.

[53] S. Axani, D. Winklehner, J. Alonso, J. M. Conrad, A high intensity $\mathrm{H}_{2}^{+}$multicusp ion source for the isotope decay-at-rest experiment, IsoDAR, Review of Scientific Instruments 87 (2) (2016) 02B704. doi:10.1063/1.4932395

[54] D. Winklehner, S. Axani, P. Bedard, J. Conrad, J. Corona, F. Hartwell, J. Smolsky, A. Tripathee, L. Waites, P. Weigel, T. Wester, M. Yampolskaya, First Commissioning Results of the Multicusp Ion Source at MIT (MIST-1) for $\mathrm{H}_{2}^{+}$, in: Proceedings of the $17^{\text {th }}$ International Conference on Ion Sources, 2017.

[55] T. Kalvas, O. Tarvainen, T. Ropponen, O. Steczkiewicz, J. Ärje, H. Clark, IBSIMU: A three-dimensional simulation software for charged particle optics, Review of Scientific Instruments 81 (2) (2010) 02B703.

[56] P. Mandal, G. Sikler, M. Mukherjee, Simulation study and analysis of a compact einzel lens-deflector for low energy ion beam Journal of Instrumentation 6 (02) (2011) P02004. URL http://stacks. iop.org/1748-0221/6/i=02/a=P02004

[57] K. R. Crandall, T. P. Wangler, PARMTEQ A beam-dynamics code fo the RFQ linear accelerator, in: Linear Accelerator and Beam Optics Codes, Vol. 177, AIP Publishing, 1988, pp. 22-28.

[58] P. Ostroumov, K. Shepard, Correction of beam-steering effects in low-velocity superconducting quarter-wave cavities, Phys. Rev. ST. Accel. Beams 4 (2001) 110101.

[59] Cst studio suite URL http://www.cst.com

[60] P. Ostroumov, K. Shepard, Design of $57.5 \mathrm{mhz} \mathrm{cw}$ rfq structure for the rare isotope accelarator facility, Pramana-Journal of Physics 59 (6) (2002) 977-988.

[61] C. Baumgarten, Transverse-longitudinal coupling by space charge in cyclotrons, Phys. Rev. ST Accel. Beams 14 (2011) 114201. doi:10.1103/PhysRevSTAB.14.114201.

URL https://link.aps.org/doi/10.1103/PhysRevSTAB.14. 114201

[62] J. Stetson, S. Adam, M. Humbel, W. Joho, T. Stammbach, THE COMMISSIONING OF PSI INJECTOR 2 FOR HIGH INTENSITY, HIGH QUALITY BEAMS, in: Proceedings of the 13th International Conference on Cyclotrons and their Applications, 1992.

[63] T. Stammbach, S. Adam, T. Blumer, D. George, A. Mezger, P. Schmelzbach, P. Sigg, The PSI $2 \mathrm{~mA}$ beam and future applications, in: AIP Conference Proceedings, Vol. 600, AIP, 2001, pp. $423-427$.

[64] A. Adelmann, A. Gsell, C. K. (PSI), Y. I. (IBM), S. R. (LANL), Y. Bi, C. Wang, J. Y. (CIAE), H. Z. T. University), S. Sheehy, C. R. (RAL), C. M. (Cornell), The OPAL (Object Oriented Parallel Accelerator Library) Framework, Tech. Rep. PSI-PR08-02, Paul Scherrer Institut ((2008-2015)).

[65] D. Campo, J. Alonso, W. Barletta, L. Bartoszek, A. Calanna, J. Conrad, M. Toups, M. Shaevitz, High intensity compact cyclotron for IsoDAR experiment, in: Proceedings of Cyclotrons, Vancouver, Canada, 2013.

(1) URL http://epaper.kek.jp/CYCLOTRONS2013/papers/ weppt030.pdf

[66] A. Calanna, D. Campo, J. J. Yang, L. Calabretta, D. Rifuggiato, M. M. Maggiore, L. A. C. Piazza, A. Adelmann, A Compact High Intensity Cyclotron Injector for DAEdALUS Experiment in: Proceedings, 3rd International Conference on Particle accelerator (IPAC 2012): New Orleans, USA, May 2-25, 2012, Vol. C1205201, 2012, pp. 424-426.

URL http://accelconf.web.cern.ch/AccelConf/IPAC2012/ 
papers/moppd027.pdf

[67] D. Toprek, Theory of the central ion trajectory in the spiral inflector, Nuclear Instruments and Methods in Physics Research Section A: Accelerators, Spectrometers, Detectors and

11 Associated Equipment 440 (2) (2000) 285 - 295. doi:https: //doi.org/10.1016/S0168-9002(99)00859-1

URL http://www.sciencedirect.com/science/article/pii/ S0168900299008591

[68] P. Weigel, Design and Simulation of the IsoDAR RFQ Direct Injection System and Spiral Inflector in: Meeting of the APS Division of Particles and Fields (DPF 2017) Batavia, Illinois, USA, July 31-August 4, 2017, 2017. arXiv:1710.00441

URL https://inspirehep.net/record/1628169/files/arXiv: 1710.00441.pdf

[69] W. Smigaj, T. Betcke, S. Arridge, J. Phillips, M. Schweiger, Solving boundary integral problems with bem++, ACM Transactions on Mathematical Software (TOMS) 41 (2) (2015) 6.

[70] G. D'Agostino, The central region of the test bench cyclotron for IsoDAR experiment, submitted to Nuovo Cimento.

[71] R. S. Lester, Report of the Committee on the Future of the MIT Nuclear Reactor Laboratory, Tech. rep., Massachusetts Institute of Technology (2015). 